\title{
Dirichlet series associated with polynomials
}

by

\author{
Manfred Peter (Freiburg)
}

1. Introduction. Let

$$
P\left(X_{1}, \ldots, X_{r}\right)=\sum_{\underline{\alpha} \in \mathbb{N}_{0}^{r}} c_{\underline{\alpha}} \underline{X}^{\underline{\alpha}} \in \mathbb{C}\left[X_{1}, \ldots, X_{r}\right]
$$

be a polynomial in $r$ variables with $\Re c_{\underline{\alpha}}>0$ if $c_{\underline{\alpha}} \neq 0$ and with $\operatorname{deg}_{X_{\varrho}} P \geq 1$ for each $1 \leq \varrho \leq r$. Let

$$
\varrho(P):=\max _{\underline{\alpha}}\left|\arg c_{\underline{\alpha}}\right|, \quad d(P):=\max \left\{\alpha_{1}+\ldots+\alpha_{r} \mid \underline{\alpha} \in \mathbb{N}_{0}^{r}, \quad c_{\underline{\alpha}} \neq 0\right\} .
$$

Let $0 \leq \Theta<\pi / 2$ and $\varrho(P)+d(P) \Theta<\pi / 2$. Let $\left(\lambda_{\varrho n}\right)_{n \geq 1}, 1 \leq \varrho \leq r$, be sequences in

$$
S_{\Theta}:=\left\{z \in \mathbb{C} \backslash \mathbb{R}_{0}^{-}|| \arg z \mid \leq \Theta\right\}
$$

with $\lim _{n \rightarrow \infty}\left|\lambda_{\varrho n}\right|=\infty$ and $\left(a_{n}^{(\varrho)}\right)_{n \geq 1}, 1 \leq \varrho \leq r$, sequences in $\mathbb{C}$. The Dirichlet series $S(s)$ is formally defined by

$$
S(s):=\sum_{n_{1}, \ldots, n_{r} \geq 1} \frac{a_{n_{1}}^{(1)} \ldots a_{n_{r}}^{(r)}}{P\left(\lambda_{1 n_{1}}, \ldots, \lambda_{r n_{r}}\right)^{s}} .
$$

Let $\sigma_{\mathrm{a}}(T) \in[-\infty, \infty]$ be the abscissa of absolute convergence of any Dirichlet series $T$. Define the auxiliary Dirichlet series

$$
S_{\varrho}(s):=\sum_{n \geq 1} \frac{a_{n}^{(\varrho)}}{\lambda_{\varrho n}^{s}}, \quad 1 \leq \varrho \leq r .
$$

In this paper the following questions are investigated:

(1) Under what conditions on the $S_{\varrho}$ is $\sigma_{\mathrm{a}}(S)$ finite and can the holomorphic function $S(s)$ be continued analytically beyond the half plane $\Re s>$ $\sigma_{\mathrm{a}}(S)$ ?

(2) Where are the singularities of $S(s)$ located and of what kind are they?

1991 Mathematics Subject Classification: 11M41, 11N45. 
(3) Is there an asymptotic formula for the summatory function of the coefficients of $S(s)$ ?

Let $\mathcal{K}$ be the set of all holomorphic functions $f:\left\{\Re s>\sigma_{f}\right\} \rightarrow \mathbb{C}$ with the properties: $f$ can be continued meromorphically to $\mathbb{C}$, has only a finite number of poles in each strip $a \leq \Re s \leq b$ and for each $\varepsilon>0$,

$$
|f(s)| \ll_{a, b, \varepsilon} e^{\varepsilon|\Im s|} \quad(a \leq \Re s \leq b,|\Im s| \rightarrow \infty) .
$$

The following two theorems are long since known.

Theorem 1.1. If $\sigma_{\mathrm{a}}\left(S_{\varrho}\right)<\infty$ and $S_{\varrho} \in \mathcal{K}$ for $1 \leq \varrho \leq r$, then $\sigma_{\mathrm{a}}(S)<\infty$ and $\Gamma S \in \mathcal{K}$.

Mellin [7], p. 23, proved this only for $\Theta=0$. The same proof works for the general situation. In [8], p. 61, Mellin remarks that even the following theorem holds.

TheOREM 1.2. If the coefficients of $P$ are real, $\sigma_{\mathrm{a}}\left(S_{\varrho}\right)<\infty$ and $S_{\varrho} \in \mathcal{K}$ for $1 \leq \varrho \leq r$, then $\sigma_{\mathrm{a}}(S)<\infty$ and $S \in \mathcal{K}$.

The proof is the same as for Theorem 1.1 with some obvious changes.

Under the strong assumption of ellipticity of $P$ more precise conclusions about the location and order of the poles of $S(s)$ can be drawn (Mahler [6]).

In the special case $P \in \mathbb{R}\left[X_{1}, \ldots, X_{r}\right], a_{n}^{(\varrho)}=\xi_{\varrho}^{n}, \lambda_{\varrho n}=n$, with $\left|\xi_{\varrho}\right|=1$, $\xi_{\varrho} \neq 1$ for $1 \leq \varrho \leq r, n \in \mathbb{N}$, the function $S(s)$ can be continued to an entire function and the numbers $S(-k), k \in \mathbb{N}_{0}$, can be computed explicitly (Cassou-Noguès [1]).

In the special case $S_{1}=\ldots=S_{r}=\zeta$ the method of Sargos [9], [10] gives a much sharper result than the method of Mellin does.

Another class of polynomials for the same $S_{\varrho}$ is investigated by Lichtin [4], [5]. This class is not defined by a condition on the coefficients of its members but by a growth condition.

In this paper Sargos' method is applied to the more general Dirichlet series (1.1). First the following sharpened version of Theorem 1.1 is obtained. Let polord ${ }_{w} S$ denote the order of the pole $w$ of $S$.

Theorem 1.3. Assume $\varrho(P)+d(P) \Theta<\pi / 2, \alpha>0$ with $\sum_{n>1}\left|\lambda_{\varrho n}\right|^{-\alpha}$ $<\infty, \sigma_{\mathrm{a}}\left(S_{\varrho}\right)<\infty$ and let $S_{\varrho}(w)$ be meromorphic on $\mathbb{C}$ for $1 \leq \varrho \leq r$. Assume that $S_{\varrho}(w)$ has only a finite number of poles in each strip

$$
B\left(\sigma_{1}, \sigma_{2}\right):=\left\{w \in \mathbb{C} \mid \sigma_{1} \leq \Re w \leq \sigma_{2}\right\} \quad\left(-\infty<\sigma_{1}<\sigma_{2}<\infty\right)
$$

and that for each $\varepsilon>0$,

$$
\left|S_{\varrho}(w)\right| \ll_{\sigma_{1}, \sigma_{2}, \varepsilon} e^{(\Theta+\varepsilon)|\Im w|}, \quad w \in B\left(\sigma_{1}, \sigma_{2}\right),|\Im w| \rightarrow \infty .
$$

Then $\sigma_{\mathrm{a}}(S)<\infty, S(w)$ can be continued meromorphically to $\mathbb{C}$, has only a finite number of poles in each vertical strip of finite width and there are 
linear forms

$$
L_{m}\left(\left(X_{\varrho}\right)_{\varrho \in I_{m}}\right), \quad I_{m} \subseteq\{1, \ldots, r\}, 1 \leq m \leq M,
$$

with coefficients in $\mathbb{N}_{0}$ which depend effectively on $P$ and effectively computable constants $N \in \mathbb{N}, c_{1}, \ldots, c_{4}>0$ so that the poles of $S(w)$ lie in the set

$$
\begin{gathered}
\left\{N^{-1}\left(a+L_{m}\left(\left(w_{\varrho}\right)_{\varrho \in I_{m}}\right)\right) \mid 1 \leq m \leq M, w_{\varrho} \text { a pole of } S_{\varrho}\right. \text { for each } \\
\left.\varrho \in I_{m}, a \in \mathbb{Z}, \Re\left(a+L_{m}\left(\left(w_{\varrho}\right)_{\varrho \in I_{m}}\right)\right) \leq c_{1} \min \left\{\Re w_{\varrho} \mid \varrho \in I_{m}\right\}+c_{2}\right\} .
\end{gathered}
$$

The order of a pole $w_{0} \in \mathbb{C}$ of $S(w)$ can be estimated by

$$
\begin{array}{r}
r+\max \left\{\sum_{\varrho \in I_{m}}\left(\operatorname{polord}_{w_{\varrho}} S_{\varrho}-1\right) \mid 1 \leq m \leq M, a \in \mathbb{Z},\right. \\
w_{\varrho} \text { a pole of } S_{\varrho} \text { for each } \varrho \in I_{m}, w_{0}=N^{-1}\left(a+L_{m}\left(\left(w_{\varrho}\right)_{\varrho \in I_{m}}\right)\right), \\
\left.c_{3} \Re w_{0}-c_{4} \leq \min \left\{\Re w_{\varrho} \mid \varrho \in I_{m}\right\}\right\} .
\end{array}
$$

Theorem 7.8 of Jorgenson and Lang [3] states that under certain conditions from an asymptotic development for theta series

$$
\sum_{k \geq 1} a_{k} e^{-\lambda_{k} t} \text { and } \sum_{k^{\prime} \geq 1} a_{k^{\prime}}^{\prime} e^{-\lambda_{k^{\prime}}^{\prime} t} \quad \text { as } t \rightarrow 0
$$

an asymptotic development for $\sum_{k, k^{\prime} \geq 1} a_{k} a_{k^{\prime}}^{\prime} e^{-\lambda_{k} \lambda_{k^{\prime}}^{\prime} t}$ can be derived. The following theorem generalizes this situation. From [3] the following axioms for Dirichlet series $\sum_{k \geq 1} a_{k} \lambda_{k}^{-s}$ and theta functions $\Theta(t)=\sum_{k \geq 1} a_{k} e^{-\lambda_{k} t}$ are taken:

DIR1. $\Re \lambda_{k}>0$ for each $k \geq 1 ; \lim _{k \rightarrow \infty} \Re \lambda_{k}=\infty$.

DIR2. (a) There is $\sigma_{0}>0$ with $\sum_{k \geq 1}\left|a_{k}\right| \cdot\left|\lambda_{k}\right|^{-\sigma_{0}}<\infty$.

(b) There is $\sigma_{1}>0$ with $\sum_{k \geq 1}\left|\lambda_{k}\right|^{-\sigma_{1}}<\infty$.

DIR3. There is $\varepsilon>0$ with $\lambda_{k} \in S(\pi / 2-\varepsilon)$ for each $k \geq 1$.

AS1. For each $C, t_{0}>0$ there are $N \in \mathbb{N}$ and $K>0$ with

$$
\left|\Theta(t)-Q_{N}(t)\right| \leq K e^{-C t} \text { for } t \geq t_{0}\left(Q_{N}(t):=\sum_{k=1}^{N-1} a_{k} e^{-\lambda_{k} t}\right) \text {. }
$$

$\mathrm{AS2}$. There are sequences $\mathcal{P}=\left(p_{n}\right)_{n \geq 0}$ in $\mathbb{C}$ and $\left(B_{p_{n}}\right)_{n \geq 0}$ in $\mathbb{C}[X]$ with the properties:

- $\Re p_{n} \uparrow \infty$ as $n \rightarrow \infty$,

- for each $q \in \mathcal{P}$,

$$
\Theta(t)-\sum_{p: \Re p<\Re q} B_{p}(\log t) t^{p}=O_{q}\left(t^{\Re q}|\log t|^{m(q)}\right)
$$

as $t \rightarrow 0\left(m(q):=\max _{p: \Re p=\Re q} \operatorname{deg} B_{p}\right)$.

AS3. For each $\delta>0$ there are $\alpha, C>0$ with $\left|\Theta(t)-Q_{N}(t)\right| \leq C t^{-\alpha}$ for each $N \in \mathbb{N}, 0<t \leq \delta$. 
As an application of Theorem 1.3 one derives

Theorem 1.4. Assume $c_{\alpha} \in \mathbb{R}$ for each $\underline{\alpha} \in \mathbb{N}_{0}^{r}$ and $d(P) \Theta<\pi / 2$. For $1 \leq \varrho \leq r$ let $S_{\varrho}$ satisfy axiom DIR2. Let the theta series $\Theta_{\varrho}(t):=$ $\sum_{k \geq 1} a_{k}^{(\varrho)} e^{-\lambda_{\varrho k} t}, t>0$, satisfy the axioms AS1-AS3. Then $S_{\varrho}$ can be continued meromorphically to $\mathbb{C}$. Assume that in each vertical strip $B\left(\sigma_{1}, \sigma_{2}\right)$ and for each $\varepsilon>0$ we have the estimate

$$
\left|S_{\varrho}(w)\right| \ll_{\sigma_{1}, \sigma_{2}, \varepsilon} e^{\varepsilon|\Im w|} \quad \text { as }|\Im w| \rightarrow \infty .
$$

Then the theta series $\Theta(t):=\sum_{k_{1}, \ldots, k_{r} \geq 1} a_{k_{1}}^{(1)} \ldots a_{k_{r}}^{(r)} e^{-P\left(\lambda_{1 k_{1}}, \ldots, \lambda_{r k_{r}}\right) t}, t>0$, satisfies the axioms $\mathrm{AS} 1-\mathrm{AS} 3$. If $\Theta_{\varrho}(t) \sim \sum_{p \in \mathcal{P}_{o}} B_{p}^{(\varrho)}(\log t) t^{p}$ is the asymptotic development of $\Theta_{\varrho}$ at 0 according to AS2 then the asymptotic development $\Theta(t) \sim \sum_{p \in \mathcal{P}} B_{p}(\log t) t^{p}$ satisfies

$$
\begin{array}{r}
\mathcal{P} \subseteq\left\{N^{-1}\left(a+L_{m}\left(\left(p_{\varrho}\right)_{\varrho \in I_{m}}\right)\right) \mid 1 \leq m \leq M,\right. \\
\quad p_{\varrho} \in \mathcal{P}_{\varrho} \text { for each } \varrho \in I_{m}, a \in \mathbb{Z}, \\
\left.\Re\left(a+L_{m}\left(\left(p_{\varrho}\right)_{\varrho \in I_{m}}\right)\right) \geq c_{1} \max \left\{\Re p_{\varrho} \mid \varrho \in I_{m}\right\}-c_{2}\right\}, \\
\operatorname{deg} B_{p} \leq r-1+\varepsilon(p)+\max \left\{\sum_{\varrho \in I_{m}}\left(\operatorname{deg} B_{p_{\varrho}}^{(\varrho)}-\varepsilon\left(p_{\varrho}\right)\right) \mid 1 \leq m \leq M,\right. \\
p_{\varrho} \in \mathcal{P}_{\varrho} \text { and } \operatorname{deg} B_{p_{\varrho}}^{(\varrho)} \geq \varepsilon\left(p_{\varrho}\right) \text { for each } \varrho \in I_{m}, a \in \mathbb{Z}, \\
\left.p=N^{-1}\left(a+L_{m}\left(\left(p_{\varrho}\right)_{\varrho \in I_{m}}\right)\right), c_{3} \Re p+c_{4} \geq \max \left\{\Re p_{\varrho} \mid \varrho \in I_{m}\right\}\right\}
\end{array}
$$

with $\varepsilon(p)=1$ for $p \in \mathbb{N}_{0}$ and $\varepsilon(p)=0$ otherwise. The $L_{\varrho}$ are as in Theorem 1.3 and $c_{1}, \ldots, c_{4}>0$.

In contrast to Mellin's method the method of Sargos can also be applied to situations in which the Dirichlet series $S_{\varrho}(s)$ have an infinite number of poles in a vertical strip of finite width. Then maybe $S(s)$ cannot be continued analytically beyond its half plane of absolute convergence but the behaviour of $S(s)$ when $s$ approaches its boundary can be analyzed and therefore a Tauberian theorem can be applied. An example for this situation is

Theorem 1.5. Assume $c_{\underline{\alpha}} \in \mathbb{R}$ for each $\underline{\alpha} \in \mathbb{N}_{0}^{r}$. Then

$$
\sum_{\substack{\left(n_{1}, \ldots, n_{r}\right) \in \mathbb{N}^{r}: \\ P\left(n_{1}, \ldots, n_{r}\right) \leq x}} \Lambda\left(n_{1}\right) \ldots \Lambda\left(n_{r}\right)=\left(C+O\left((\log \log x)^{-1}\right)\right) x^{\sigma_{\mathrm{a}}}(\log x)^{\omega} \quad(x \rightarrow \infty)
$$

with constants $C, \sigma_{\mathrm{a}}>0, \omega \in \mathbb{N}_{0}, 0 \leq \omega \leq r-1$.

Let $c>1$. In [7], p. 24, Mellin mentioned the series

$$
S(s)=\sum_{n_{1}, \ldots, n_{r} \geq 0} P\left(c^{n_{1}}, \ldots, c^{n_{r}}\right)^{-s},
$$


about which he explicitly stated that his method cannot be applied to it. But Sargos' method can be applied and gives

THEOREM 1.6. The holomorphic function which is defined by (1.7) in the right half plane $\Re s>0$ can be continued meromorphically to $\mathbb{C}$. Its poles lie in $N^{-1}\left(-\mathbb{N}_{0}+2 \pi i(\log c)^{-1} \mathbb{Z}\right)$ for a suitable $N \in \mathbb{N}$ and have order $\leq r$.

Corollary 1.7. Assume $c_{\underline{\alpha}} \in \mathbb{R}$ for each $\underline{\alpha} \in \mathbb{N}_{0}^{r}$ and $c>1$. Then there are constants $C>0$ and $\omega \in \bar{N}, \omega \leq r$, so that as $x \rightarrow \infty$,

$\#\left\{\left(n_{1}, \ldots, n_{r}\right) \in \mathbb{N}_{0}^{r} \mid P\left(c^{n_{1}}, \ldots, c^{n_{r}}\right) \leq x\right\}=\left(C+O\left((\log \log x)^{-1}\right)\right) \log ^{\omega} x$.

In this case the meromorphic continuation to $\mathbb{C}$ is possible because the integral linear combinations of the poles $2 \pi i n(\log c)^{-1}, n \in \mathbb{Z}$, of $S_{\varrho}(w)=$ $\left(1-c^{-w}\right)^{-1}$ have no accumulation point in $\mathbb{C}$. For the same reason the following theorem holds.

TheOREM 1.8. The holomorphic function which is defined by

$$
S(s):=\sum_{n_{1}, \ldots, n_{r} \geq 1} \frac{\Lambda\left(n_{1}\right)}{P\left(n_{1}, \ldots, n_{r}\right)^{s}}
$$

in a right half plane can be continued meromorphically to $\mathbb{C}$. Its poles lie in $\left\{N^{-1}\left(k+c_{m} \varrho\right) \mid k \in \mathbb{Z}, k \leq c, \varrho\right.$ a nontrivial zero of $\left.\zeta(s), 1 \leq m \leq M\right\}$ for suitable $N, c \in \mathbb{N}, c_{1}, \ldots, c_{M} \in \mathbb{N}_{0}$, and have order $\leq r$.

2. Some auxiliary theorems. The following transformations are an essential tool in [9]: For $\lambda=\left(\lambda_{j k}\right) \in \mathbb{N}_{0}^{r \times r}$ with $\operatorname{det} \underline{\underline{\lambda}} \neq 0$ define $\omega=$ $\omega(\underline{\underline{\lambda}}):(1, \infty)^{r} \rightarrow(1, \infty)^{r}$ by $\omega \underline{x}:=\left(x_{1}^{\lambda_{1 j}} \ldots x_{r}^{\lambda_{r j}}\right)_{1 \leq j \leq r}$. It is a $C^{\infty}$-function and has the functional determinant $J \omega(\underline{x})=\operatorname{det} \underline{\underline{\lambda}} \prod_{j=1}^{r} x_{j}^{-1} \prod_{k=1}^{r} x_{k}^{\lambda_{k j}}$. Let $\Omega:=\left\{\omega(\underline{\underline{\lambda}}) \mid \underline{\underline{\lambda}} \in \mathbb{N}_{0}^{r \times r}, \operatorname{det} \underline{\underline{\lambda}} \neq 0\right\}$. If $Q(\underline{X})=\sum_{\underline{\alpha}} d_{\underline{\alpha}} \underline{X} \underline{\alpha} \in \mathbb{C}[\underline{X}]$, then $Q_{\omega}(\underline{X}):=\sum_{\underline{\alpha}} d_{\underline{\alpha}} \underline{X} \underline{\underline{\lambda}} \underline{\alpha}$ is a polynomial with $Q_{\omega}(\underline{x})=Q(\omega(\underline{x}))$ for each $\underline{x} \in(1, \infty)^{r}$. One says that $Q$ has a greatest monomial $d_{\beta} \underline{X}^{\beta}$ if $d_{\beta} \neq 0$ and $\underline{\alpha} \leq \underline{\beta}$ for each $\underline{\alpha}$ with $d_{\underline{\alpha}} \neq 0$. Here $\underline{\alpha} \leq \underline{\beta}$ is shorthand for $\alpha_{j} \leq \bar{\beta}_{j}$ for each $1 \leq \bar{j} \leq r$.

The following theorem is crucial for Sargos' method.

TheOREM 2.1 ([9], Theorem 2.1). For each $P \in \mathbb{C}[\underline{X}]$ there are $\omega_{1}, \ldots, \omega_{m}$ $\in \Omega$ with the following properties:

(1) $(1, \infty)^{r}$ is up to sets of Lebesgue measure zero the disjoint union of the sets $\omega_{\mu}\left((1, \infty)^{r}\right), 1 \leq \mu \leq m$.

(2) $P_{\omega_{\mu}}$ has a greatest monomial for each $1 \leq \mu \leq m$.

(3) $\omega_{1}, \ldots, \omega_{m}$ depend only upon $\operatorname{supp} P:=\left\{\underline{\alpha} \in \mathbb{N}_{0}^{r} \mid \underline{X} \underline{\alpha}\right.$ appears in $\left.P\right\}$. 
As in [9], $S(s)$ is represented as an integral with the help of the residue theorem. Therefore functions $G_{\varrho}(z), 1 \leq \varrho \leq r$, on $S_{\Theta}$ are needed which have poles of order 1 and residues $\left(a_{n}^{(\varrho)}\right)_{n \geq 1}$ exactly at the points $\left(\lambda_{\varrho n}\right)_{n \geq 1}$ and for which the asymptotic behaviour as $|z| \rightarrow \infty$ is known on each ray $z=r e^{i \phi}, \Theta<|\phi|<\pi / 2$. In [9], $G_{\varrho}(z)=(e(z)-1)^{-1}$ with $e(z):=\exp (2 \pi i z)$ is used; in this paper $G_{\varrho}(z)$ is constructed by a Mittag-Leffler series. The asymptotic development is obtained with a method of Mellin.

Lemma 2.2. Assume $0 \leq \Theta<\pi$ and let $\left(\lambda_{n}\right)_{n \geq 1}$ be a sequence in $S_{\Theta}$ with $\lim _{n \rightarrow \infty}\left|\lambda_{n}\right|=\infty,\left(a_{n}\right)_{n \geq 1}$ a sequence in $\mathbb{C}$ and $p \in \mathbb{N}_{0}$. Assume that the Dirichlet series $T(w):=\sum_{n \geq 1} a_{n} \lambda_{n}^{-w}$ is absolutely convergent for $\Re w \geq p+1$. Then the series

$$
M T(z):=\sum_{n \geq 1} a_{n}\left(\left(z+\lambda_{n}\right)^{-1}-\sum_{k=0}^{p-1} \lambda_{n}^{-(k+1)}(-z)^{k}\right)
$$

is uniformly convergent on each compact subset of $\mathbb{C}$ after omitting finitely many summands. For each $z \in \mathbb{C} \backslash \mathbb{R}_{0}^{-}$with $|\arg z|<\pi-\Theta$ and $p+1<a<$ $p+2$, we have the identity

$$
M T(z)=(-z)^{p} T(p+1)+\frac{1}{2 \pi i} \int_{a-i \infty}^{a+i \infty} \frac{\pi}{\sin \pi w} T(w) z^{w-1} d w .
$$

Proof. The following is a slight adaptation of the proof in [7], $\S 1$. The first part of the theorem is standard for Mittag-Leffler series. For each $a \in$ $\mathbb{R}^{+} \backslash \mathbb{N}$ and $z \in \mathbb{C} \backslash \mathbb{R}_{0}^{-}$, define

$$
I(a, z):=\frac{1}{2 \pi i} \int_{a-i \infty}^{a+i \infty} \frac{\pi}{\sin \pi w} z^{w-1} d w .
$$

For each $w \in \mathbb{C}$ with $|\Im w| \geq \delta>0$, the estimate $(\sin \pi w)^{-1} \ll_{\delta} e^{-\pi|\Im w|}$ holds. Therefore the integral is absolutely and uniformly convergent with respect to $z$ on each compact subset of $\mathbb{C} \backslash \mathbb{R}_{0}^{-}$. So it is holomorphic with respect to $z$. From the residue theorem it follows that

$$
I(a, z)=I(a+1, z)+(-1)^{[a]} z^{[a]} .
$$

If $z \in \mathbb{C} \backslash \mathbb{R}_{0}^{-}, a \in \mathbb{R}^{+}, \min _{n \in \mathbb{Z}}|a-n| \geq \delta>0$, then $|I(a, z)| \ll_{z, \delta}|z|^{a-1}$ uniformly in $a$. For fixed $z \in \mathbb{C} \backslash \mathbb{R}_{0}^{-}$with $|z|<1$, and $0<a<1$, it follows from (2.1) that

$I(a, z)=\sum_{n=0}^{q-1}(-1)^{n} z^{n}+I(a+q, z) \rightarrow \sum_{n \geq 0}(-1)^{n} z^{n}=(1+z)^{-1} \quad$ as $q \rightarrow \infty$, and therefore $I(a, z)=(1+z)^{-1}$. From (2.1) it follows by holomorphic 
continuation that for $z \in \mathbb{C} \backslash \mathbb{R}_{0}^{-}$and $a \in \mathbb{R}^{+} \backslash \mathbb{N}$ the identity

$$
(1+z)^{-1}+\sum_{n=1}^{[a]}(-1)^{n} z^{n-1}=\frac{1}{2 \pi i} \int_{a-i \infty}^{a+i \infty} \frac{\pi}{\sin \pi w} z^{w-1} d w
$$

holds. For $z \in \mathbb{C} \backslash \mathbb{R}_{0}^{-}$with $|\arg z|<\pi-\Theta$, and $p+1<a<p+2$, we have $|\arg z|+\left|\arg \lambda_{n}\right|<\pi$ and therefore $z \lambda_{n}^{-1} \in \mathbb{C} \backslash \mathbb{R}_{0}^{-}$for each $n \in \mathbb{N}$. By $(2.2)$ the $n$th summand in $M T(z)$ is therefore equal to

$$
\begin{aligned}
a_{n} \lambda_{n}^{-1}((1 & \left.\left.+z \lambda_{n}^{-1}\right)^{-1}+\sum_{k=1}^{p}(-1)^{k}\left(z \lambda_{n}^{-1}\right)^{k-1}\right) \\
& =a_{n} \lambda_{n}^{-1}\left((-1)^{p}\left(z \lambda_{n}^{-1}\right)^{p}+\frac{1}{2 \pi i} \int_{a-i \infty}^{a+i \infty} \frac{\pi}{\sin \pi w}\left(z \lambda_{n}^{-1}\right)^{w-1} d w\right) \\
& =(-z)^{p} a_{n} \lambda_{n}^{-(p+1)}+\frac{1}{2 \pi i} \int_{a-i \infty}^{a+i \infty} \frac{\pi}{\sin \pi w} z^{w-1} a_{n} \lambda_{n}^{-w} d w .
\end{aligned}
$$

Summation over $n$ gives the representation of $M T(z)$ after interchanging integration and summation. This is allowed because for $w=a+i t$,

$$
\begin{aligned}
\left|\frac{\pi}{\sin \pi w} z^{w-1} a_{n} \lambda_{n}^{-w}\right| & \ll e^{-\pi|t|}|z|^{a-1} e^{|t| \cdot|\arg z|}\left|a_{n}\right| \cdot\left|\lambda_{n}\right|^{-a} e^{|t| \Theta} \\
& \ll_{z, a}\left|a_{n}\right| \cdot\left|\lambda_{n}\right|^{-(p+1)} e^{-|t|(\pi-|\arg z|-\Theta)} .
\end{aligned}
$$

Under certain meromorphy conditions on $T(w)$ an asymptotic development of $M T(w)$ can be proved which is a generalization of Stirling's formula for $\Gamma^{\prime} / \Gamma(w)$.

Theorem 2.3. Assume $0 \leq \Theta<\pi$ and let $\left(\lambda_{n}\right)_{n>1}$ be a sequence in $S_{\Theta}$ with $\lim _{n \rightarrow \infty}\left|\lambda_{n}\right|=\infty$ and $\left(a_{n}\right)_{n \geq 1}$ a sequence in $\mathbb{C}$. Assume that the Dirichlet series $T(w):=\sum_{n \geq 1} a_{n} \lambda_{n}^{-w}$ has the following properties:

(1) There is $p \in \mathbb{N}_{0}$ so that $T(w)$ converges absolutely for $\Re w \geq p+1$.

(2) $T(w)$ can be continued meromorphically to $\mathbb{C}$.

(3) For each $-\infty<\sigma_{1}<\sigma_{2}<\infty$ only finitely many poles of $T(w)$ lie in $B\left(\sigma_{1}, \sigma_{2}\right)$ and for each $\varepsilon>0$,

$$
|T(w)| \ll_{\sigma_{1}, \sigma_{2}, \varepsilon} e^{(\Theta+\varepsilon)|\Im w|}, \quad w \in B\left(\sigma_{1}, \sigma_{2}\right),|\Im w| \rightarrow \infty .
$$

Then for each $b \in(-\infty, p+1) \backslash \mathbb{Z}$ for which $T(w)$ has no poles on $\Re w=b$ and for each $\varepsilon>0$ and $z \in S_{\pi-\Theta-\varepsilon}$ with $|z| \geq 1$,

$$
M T(z)=\sum_{b<\Re w_{0}<p+1} \operatorname{Res}_{w_{0}}\left(\frac{\pi}{\sin \pi w} T(w) z^{w-1}\right)+O_{b, \varepsilon}\left(|z|^{b-1}\right) .
$$


Proof. If $-\infty<\sigma_{1}<\sigma_{2}<\infty, z \in S_{\pi-\Theta-\varepsilon},|z| \geq 1$ then for $w \in$ $B\left(\sigma_{1}, \sigma_{2}\right),|\Im w| \rightarrow \infty$, we have

$$
\begin{aligned}
\left|\frac{\pi}{\sin \pi w} T(w) z^{w-1}\right| & \ll_{\sigma_{1}, \sigma_{2}, \varepsilon} e^{-\pi|\Im w|+(\Theta+\varepsilon / 2)|\Im w|}|z|^{\sigma_{2}-1} e^{|\Im w| \cdot|\arg z|} \\
& \ll_{\sigma_{1}, \sigma_{2}, \varepsilon} e^{-\varepsilon|\Im w| / 2}|z|^{\sigma_{2}-1} .
\end{aligned}
$$

Choose $a=p+3 / 2$. From Lemma 2.2 and the residue theorem,

$$
\begin{aligned}
M T(z)= & (-z)^{p} T(p+1)+\frac{1}{2 \pi i} \int_{b-i \infty}^{b+i \infty} \frac{\pi}{\sin \pi w} T(w) z^{w-1} d w \\
& +\sum_{b<\Re w_{0}<a} \operatorname{Res}_{w_{0}}\left(\frac{\pi}{\sin \pi w} T(w) z^{w-1}\right) .
\end{aligned}
$$

In $\{p+1 \leq \Re w<a\}$ the only pole of $\pi(\sin \pi w)^{-1} T(w) z^{w-1}$ lies at $p+1$ and has residue $(-1)^{p+1} T(p+1) z^{p}$. The integral in $(2.3)$ is

$$
\ll_{b, \varepsilon} \int_{-\infty}^{\infty} e^{-\pi|t|+(\Theta+\varepsilon / 2)|t|}|z|^{b-1} e^{|t| \cdot|\arg z|} d t \ll_{b, \varepsilon}|z|^{b-1} .
$$

From this the statement of the theorem follows.

LEMMA 2.4. Let the sequences $\left(\lambda_{\varrho n}\right)_{n \geq 1}$ and $\left(a_{n}^{(\varrho)}\right)_{n \geq 1}$ fulfil the assumptions of Lemma 2.2 for $1 \leq \varrho \leq r$ with a common $\Theta$ and $p$ and assume $\alpha>0$ with $\sum_{n>1}\left|\lambda_{\varrho n}\right|^{-\alpha}<\infty$ for $1 \leq \varrho \leq r$. Then there is a sequence $\left(R_{m}\right)_{m \geq 1}$ which tends monotonically to infinity and has the property:

$$
\left|M T_{\varrho}(z)\right| \ll R_{m}^{p+\alpha} \quad \text { for each }|z|=R_{m}, \quad m \geq 1,1 \leq \varrho \leq r .
$$

Proof. With $C_{\varrho}:=\sum_{n \geq 1}\left|\lambda_{\varrho n}\right|^{-\alpha}$ it follows that for each $R \geq 1$,

$$
\#\left\{n \geq 1|| \lambda_{\varrho n} \mid \leq R\right\} \leq \sum_{n \geq 1}\left(\frac{R}{\left|\lambda_{\varrho n}\right|}\right)^{\alpha}=C_{\varrho} R^{\alpha} .
$$

Consequently, for each $R \geq 1$ there is an $R^{\prime} \in[R, 2 R]$ with $\left|R^{\prime}-\right| \lambda_{\varrho n}|| \gg$ $R^{\prime 1-\alpha}$ for each $n \in \mathbb{N}, 1 \leq \varrho \leq r$, with a $\gg$-constant which is independent of $R, \varrho, n$. For $z \in \mathbb{C}$ with $|z|=R^{\prime}$ and $1 \leq \varrho \leq r$ it follows that

$$
\begin{aligned}
\left|M T_{\varrho}(z)\right| \leq & \left(\sum_{\left|\lambda_{\varrho n}\right| \leq R^{\prime} / 2}+\sum_{R^{\prime} / 2 \leq\left|\lambda_{\varrho n}\right| \leq 2 R^{\prime}}\right)\left|a_{n}^{(\varrho)}\right|\left(\frac{1}{|z|-\left|\lambda_{\varrho n}\right|}+\sum_{k=0}^{p-1} \frac{|z|^{k}}{\left|\lambda_{\varrho n}\right|^{k+1}}\right) \\
& +\sum_{\left|\lambda_{\varrho n}\right| \geq 2 R^{\prime}}\left|a_{n}^{(\varrho)}\right|\left|\frac{1}{\lambda_{\varrho n}} \sum_{k \geq p}\left(\frac{-z}{\lambda_{\varrho n}}\right)^{k}\right|
\end{aligned}
$$




$$
\begin{aligned}
& \ll \sum_{\left|\lambda_{\varrho n}\right| \leq R^{\prime} / 2}\left|a_{n}^{(\varrho)}\right|\left(\frac{1}{R^{\prime}}+\frac{1}{R^{\prime}} \sum_{k=0}^{p-1}\left(\frac{R^{\prime}}{\left|\lambda_{\varrho n}\right|}\right)^{k+1}\right) \\
& +\sum_{R^{\prime} / 2 \leq\left|\lambda_{\varrho n}\right| \leq 2 R^{\prime}}\left|a_{n}^{(\varrho)}\right|\left(R^{\prime \alpha-1}+\sum_{k=0}^{p-1} \frac{R^{\prime k}}{\left(R^{\prime} / 2\right)^{k+1}}\right) \\
& +\sum_{\left|\lambda_{\varrho n}\right| \geq 2 R^{\prime}}\left|a_{n}^{(\varrho)}\right| \frac{|z|^{p}}{\left|\lambda_{\varrho n}\right|^{p+1}} \cdot \frac{1}{1-\left|z / \lambda_{\varrho n}\right|} \\
& \ll \sum_{\left|\lambda_{\varrho n}\right| \leq R^{\prime} / 2}\left|a_{n}^{(\varrho)}\right| \frac{R^{\prime p-1}}{\left|\lambda_{\varrho n}\right|^{p}} \cdot \frac{R^{\prime}}{\left|\lambda_{\varrho n}\right|} \\
& +\sum_{R^{\prime} / 2 \leq\left|\lambda_{\varrho n}\right| \leq 2 R^{\prime}} \frac{\left|a_{n}^{(\varrho)}\right|}{\left|\lambda_{\varrho n}\right|^{p+1}}\left(2 R^{\prime}\right)^{p+1} R^{\prime \alpha-1}+R^{\prime p} \ll R^{\prime \alpha+p} .
\end{aligned}
$$

Lemma 2.5. Let $k \in \mathbb{N}_{0}$. There are polynomials $P_{k \kappa} \in \mathbb{C}[x]$ with $\operatorname{deg} P_{k \kappa}$ $\leq k-\kappa$ and with the property: If $h(z)$ is holomorphic on an open neighbourhood $U$ of $[0,1]$ in $\mathbb{C}$ which contains the closed disk around 0 with radius $\varepsilon>0$ and $\alpha \in \mathbb{C} \backslash \mathbb{Z}, \Re \alpha>-1$, then

$$
\int_{0}^{1} x^{\alpha} h(x) \log ^{k} x d x=\sum_{\kappa=0}^{k} \frac{P_{k \kappa}(e(\alpha))}{(e(\alpha)-1)^{k+1-\kappa}} \int_{I_{\varepsilon}} z^{\alpha} h(z) \log ^{\kappa} z d z .
$$

Here a slit is made in the complex plane along the positive real axis and $\log z$ is defined on $\mathbb{C} \backslash \mathbb{R}_{0}^{+}$where arg $z$ takes values from 0 to $2 \pi$. $I_{\varepsilon}$ consists of the path from 1 to $\varepsilon$ above the slit, the circle around 0 with radius $\varepsilon$ which starts at $\varepsilon$ and is run through in the positive direction and the path from $\varepsilon$ to 1 below the slit.

Proof. The case $k=0$ is contained in Lemma 4.1 of [9]. The general case follows by taking the $k$ th derivative with respect to $\alpha$.

LEMMA 2.6. The series

$$
G(z):=\sum_{n \geq 1}\left(\frac{\Lambda(n)}{z+n}-\frac{\Lambda(n)}{n}\right)
$$

defines a meromorphic function on $\mathbb{C}$ whose poles are at $-n, n \in \mathbb{N}$, are simple and have the respective residues $\Lambda(n)$. For each $b \in \mathbb{R}^{-} \backslash \mathbb{Z}, z \in \mathbb{C} \backslash \mathbb{R}_{0}^{-}$ with $|z| \geq 1$ and $|\arg z| \leq \pi-\varepsilon, \varepsilon>0$, we have the asymptotic formula

$$
G(z)=-\sum_{\varrho} \frac{\pi}{\sin \pi \varrho} z^{\varrho-1}+\sum_{[b]+1 \leq n \leq 1} z^{n-1}\left(a_{n} \log z+b_{n}\right)+O_{b, \varepsilon}\left(|z|^{b-1}\right)
$$


where $\varrho$ runs through the nontrivial zeros of $\zeta(s), a_{n}, b_{n}$ are complex constants and $\log z$ is the principal branch of the logarithm. Furthermore, for $z \in \mathbb{C} \backslash \mathbb{R}_{0}^{-}$with $|\arg z| \leq \pi-\varepsilon, \varepsilon>0,|z| \geq e^{2}$, we have

$$
G(z)=a_{1} \log z+b_{1}+R(z), \quad R(z)=O_{\varepsilon}\left(e^{-c \log |z| / \log \log |z|}\right)
$$

with a constant $c>0$. For $z \in \mathbb{C}$ with $\min _{n \in \mathbb{N}}|z+n| \geq 1 / 2$,

$$
G(z) \ll \log ^{2}(|z|+2) .
$$

Proof. According to Lemma 2.2 with $\Theta=0, p=1$ and $T(w)=$ $-\zeta^{\prime} / \zeta(w)$ the first part of the statement holds for $G=M\left(-\zeta^{\prime} / \zeta\right)$. For $z \in \mathbb{C} \backslash \mathbb{R}_{0}^{-}$and $a=5 / 2$,

$$
G(z)=z \frac{\zeta^{\prime}}{\zeta}(2)-\frac{1}{2 \pi i} \int_{a-i \infty}^{a+i \infty} \frac{\pi}{\sin \pi w} \cdot \frac{\zeta^{\prime}}{\zeta}(w) z^{w-1} d w .
$$

Let $b \in \mathbb{R}^{-} \backslash \mathbb{Z}$ and in addition $|z| \geq 1,|\arg z| \leq \pi-\varepsilon$. Choose a sequence $\left(T_{m}\right)_{m \geq 1}$ which converges monotonically to infinity so that for each $b \leq$ $\Re w \leq a, \Im w= \pm T_{m}$, the estimate $\left|\zeta^{\prime} / \zeta(w)\right| \ll_{a, b} \log ^{2} T_{m}$ holds (Davenport [2], p. 112, and the functional equation). From the residue theorem it follows that

$$
\begin{aligned}
G(z)= & z \frac{\zeta^{\prime}}{\zeta}(2)-\frac{1}{2 \pi i} \int_{b-i T_{m}}^{b+i T_{m}} \frac{\pi}{\sin \pi w} \cdot \frac{\zeta^{\prime}}{\zeta}(w) z^{w-1} d w \\
& +O_{\varepsilon}\left(e^{-\pi T_{m}} \log ^{2} T_{m}|z|^{a-1} e^{T_{m}|\arg z|}\right)-\sum_{\varrho:|\Im \varrho|<T_{m}} \frac{\pi}{\sin \pi \varrho} z^{\varrho-1} \\
& -\sum_{[b]+1 \leq n \leq 2} \operatorname{Res}_{w=n}\left(\frac{\pi}{\sin \pi w} \cdot \frac{\zeta^{\prime}}{\zeta}(w) z^{w-1}\right) .
\end{aligned}
$$

For each fixed $z$ the $O$-term converges towards 0 as $m \rightarrow \infty$. For each nontrivial zero $\varrho$ of $\zeta$,

$$
\left|\frac{\pi}{\sin \pi \varrho} z^{\varrho-1}\right| \ll e^{-|\Im \varrho|(\pi-|\arg z|)}|z|^{\Re \varrho-1},
$$

and consequently the sum over $\varrho$ is absolutely convergent as $m \rightarrow \infty$. By letting $m \rightarrow \infty,(2.4)$ follows with error term

$$
\begin{aligned}
-\frac{1}{2 \pi i} \int_{b-i \infty}^{b+i \infty} \frac{\pi}{\sin \pi w} & \cdot \frac{\zeta^{\prime}}{\zeta}(w) z^{w-1} d w \\
& \ll_{b} \int_{-\infty}^{\infty} e^{-\pi|t|} \log (|t|+2)|z|^{b-1} e^{|\arg z| \cdot|t|} d t \ll_{b, \varepsilon}|z|^{b-1} .
\end{aligned}
$$

If $|\arg z| \leq \pi-\varepsilon$ and $|z| \geq e^{2}$, then choose $b=-1 / 2$. For each $\varrho$ it follows from Davenport [2], p. 89, that $\Re \varrho \leq 1-c \log ^{-1}(|\Im \varrho|+2)$ with a constant 
$c>0$. With $R:=\log (|z|+2) \geq 2$ we have

$$
\begin{aligned}
\left|\sum_{\varrho} \frac{\pi}{\sin \pi \varrho} z^{\varrho-1}\right| & \ll \sum_{\varrho} \exp \left(-|\Im \varrho|(\pi-(\pi-\varepsilon))-\frac{c R}{\log (|\Im \varrho|+2)}\right) \\
& \ll \sum_{|\Im \varrho| \leq R} \exp \left(-|\Im \varrho| \varepsilon-\frac{c R}{\log R}\right) \\
& +\sum_{|\Im \varrho|>R} \exp \left(-\frac{\varepsilon}{2}|\Im \varrho|-\frac{\varepsilon}{2} R\right) \\
& \ll \varepsilon \exp \left(-\frac{c R}{\log R}\right) .
\end{aligned}
$$

Substituting into (2.4) gives (2.5).

For the last part of the statement assume $|z| \geq 2$ with $\min _{n \in \mathbb{N}}|z+n| \geq$ $1 / 2$. Then

$$
|G(z)| \leq|z| \sum_{n \geq 1} \Lambda(n)(|z+n| n)^{-1} .
$$

On the intervals $[1,|z| / 2],(|z| / 2,|z|-1],(|z|-1,|z|+1],(|z|+1,2|z|],(2|z|, \infty)$ the summands can be estimated by $O\left(\Lambda(n)(n|z|)^{-1}\right), O\left(\Lambda(n)(n(|z|-n))^{-1}\right)$, $O\left(\Lambda(n) n^{-1}\right), O\left(\Lambda(n)(n(n-|z|))^{-1}\right), O\left(\Lambda(n) n^{-2}\right)$ respectively. Partial summation ends the proof of the lemma.

Lemma 2.7. For each $1 \leq \varrho \leq r$ let $\left(\lambda_{\varrho n}\right)_{n>1}$ be a sequence in $\mathbb{R}^{+}$which converges monotonically towards infinity, $\left(a_{n}^{(\varrho)}\right)_{n \geq 1}$ be a sequence in $\mathbb{R}_{0}^{+}$, and $\sigma^{(\varrho)}>0$ with

$$
\sum_{y \leq \lambda_{\varrho n}<2 y} a_{n}^{(\varrho)} \asymp y^{\sigma^{(\varrho)}} \quad \text { as } y \rightarrow \infty .
$$

Let $P \in \mathbb{R}[\underline{X}]$ have nonnegative coefficients and $\operatorname{deg}_{X_{e}} P \geq 1$ for each $1 \leq$ $\varrho \leq r$. Then

$$
S(s)=\sum_{n_{1}, \ldots, n_{r} \geq 1} \frac{a_{n_{1}}^{(1)} \ldots a_{n_{r}}^{(r)}}{P\left(\lambda_{1 n_{1}}, \ldots, \lambda_{r n_{r}}\right)^{s}}
$$

has the abscissa of (absolute) convergence

$$
\sigma_{\mathrm{a}}(S)=\min \left\{\sigma>0 \mid P(\underline{x})^{\sigma} \gg_{\sigma} x_{1}^{\sigma^{(1)}} \ldots x_{r}^{\sigma^{(r)}} \text { for } \underline{x} \in(1, \infty)^{r}\right\}
$$

and $S(s)$ is divergent for $s=\sigma_{\mathrm{a}}(S)$.

Proof. Let $y_{1}, \ldots, y_{r} \geq 1$ and $\sigma>0$. Then for $y_{\varrho} \leq x_{\varrho} \leq 2 y_{\varrho}, 1 \leq \varrho \leq r$,

$$
P\left(y_{1}, \ldots, y_{r}\right) \leq P\left(x_{1}, \ldots, x_{r}\right) \leq P\left(2 y_{1}, \ldots, 2 y_{r}\right) \leq 2^{d(P)} P\left(y_{1}, \ldots, y_{r}\right)
$$


and therefore

$$
\begin{aligned}
\sum_{\substack{y_{\varrho} \leq \lambda_{\varrho n_{\varrho}<2 y_{\varrho}} \leq \varrho \leq r \\
1 \leq \varrho \leq r}} \frac{a_{n_{1}}^{(1)} \ldots a_{n_{r}}^{(r)}}{P\left(\lambda_{1 n_{1}}, \ldots, \lambda_{r n_{r}}\right)^{\sigma}} & { }_{\sigma} P\left(y_{1}, \ldots, y_{r}\right)^{-\sigma} y_{1}^{\sigma^{(1)}} \ldots y_{r}^{\sigma^{(r)}} \\
& \asymp_{\sigma} \int_{y_{1}}^{2 y_{1}} \ldots \int_{y_{r}}^{2 y_{r}} \frac{x_{1}^{\sigma^{(1)}}-1 \ldots x_{r}^{\sigma^{(r)}}-1}{P\left(x_{1}, \ldots, x_{r}\right)^{\sigma}} d x_{1} \ldots d x_{r} .
\end{aligned}
$$

Summation over $y_{\varrho}=2^{l_{\varrho}}, l_{\varrho} \in \mathbb{N}_{0}, 1 \leq \varrho \leq r$, gives

$$
\sum_{n_{1}, \ldots, n_{r} \geq 1} \frac{a_{n_{1}}^{(1)} \ldots a_{n_{r}}^{(r)}}{P\left(\lambda_{1 n_{1}}, \ldots, \lambda_{r n_{r}}\right)^{\sigma}} \asymp_{\sigma} \int_{1}^{\infty} \ldots \int_{1}^{\infty} \frac{x_{1}^{\sigma^{(1)}-1} \ldots x_{r}^{\sigma^{(r)}}-1}{P\left(x_{1}, \ldots, x_{r}\right)^{\sigma}} d x_{1} \ldots d x_{r} .
$$

Hence the left hand side is finite if and only if the right hand side is finite. The right hand side is $Y\left(P, \sigma^{(1)}-1, \ldots, \sigma^{(r)}-1 ; \sigma\right)$ in the notation of [9]. From Theorem 3.1 and Lemma 3.2 in that paper the statement follows.

Lemma 2.8. Let $c>1$. Then $G(z):=\sum_{n>0}\left(z+c^{n}\right)^{-1}$ is a meromorphic function on $\mathbb{C}$ with simple poles at $-c^{n}, n \in \mathbb{N}_{0}$, and residue 1 respectively. For $z \in \mathbb{C} \backslash \mathbb{R}_{0}^{-}$with $|z| \geq 1,|\arg z| \leq \pi-\varepsilon, \varepsilon>0$, and $b \in \mathbb{R}^{-} \backslash \mathbb{Z}$, we have the asymptotic formula

$$
\begin{aligned}
G(z)= & \frac{\log z}{z \log c}+\frac{1}{2 z}+\sum_{[b]+1 \leq m \leq-1}(-1)^{m}\left(1-c^{-m}\right)^{-1} z^{m-1} \\
& +\frac{\pi}{z \log c} \sum_{0 \neq n \in \mathbb{Z}} \sin \left(\frac{2 \pi^{2} i n}{\log c}\right)^{-1} \exp \left(\frac{2 \pi i n \log z}{\log c}\right)+O_{b, \varepsilon}\left(|z|^{b-1}\right) .
\end{aligned}
$$

The nth summand is $\ll \exp (-2 \pi|n| \varepsilon / \log c)$. For each $n_{0} \in \mathbb{N}$ and $|z|=$ $(1+c) c^{n_{0}} / 2$

$$
|G(z)| \ll|z|^{-1} \log (|z|+2) .
$$

Proof. For $\Re w>0$ define $T(w):=\sum_{n \geq 0} c^{-n w}=\left(1-c^{-w}\right)^{-1}$. Choosing $p=0, \Theta=0, a=3 / 2$ in Lemma 2.2 it follows that for each $z \in \mathbb{C} \backslash \mathbb{R}_{0}^{-}$ with $|\arg z| \leq \pi-\varepsilon$ and $|z| \geq 1$,

$$
G(z)=T(1)+\frac{1}{2 \pi i} \int_{a-i \infty}^{a+i \infty} \frac{\pi}{\sin \pi w} \cdot \frac{z^{w-1}}{1-c^{-w}} d w .
$$

Let $b \in \mathbb{R}^{-} \backslash \mathbb{Z}$ and $T_{k}:=2 \pi(2 k+1)(2 \log c)^{-1}, k \in \mathbb{N}$. For $\sigma \in \mathbb{R}$, we have $\left|1-c^{-\left(\sigma \pm i T_{k}\right)}\right| \geq 1$. Applying the residue theorem to the rectangle with vertices $a \pm i T_{k}, b \pm i T_{k}$ and letting $k \rightarrow \infty$ gives

$$
G(z)=T(1)+\frac{1}{2 \pi i} \int_{b-i \infty}^{b+i \infty} \frac{\pi}{\sin \pi w} \cdot \frac{z^{w-1}}{1-c^{-w}} d w
$$




$$
\begin{aligned}
& +\sum_{[b]+1 \leq m \leq 1} \operatorname{Res}_{m}\left(\frac{\pi}{\sin \pi w} \cdot \frac{z^{w-1}}{1-c^{-w}}\right) \\
& +\sum_{0 \neq n \in \mathbb{Z}} \operatorname{Res}_{\frac{2 \pi i n}{\log c}}\left(\frac{\pi}{\sin \pi w} \cdot \frac{z^{w-1}}{1-c^{-w}}\right) .
\end{aligned}
$$

The integral is $\ll_{b} \int_{-\infty}^{\infty} e^{-\pi|t|}|z|^{b-1} e^{|t|(\pi-\varepsilon)} d t \ll_{b, \varepsilon}|z|^{b-1}$. For $[b]+1 \leq$ $m \leq 1, m \neq 0$, the $m$ th summand is $(-1)^{m} z^{m-1}\left(1-c^{-m}\right)^{-1}$. For $m=$ 0 it is $\log z(z \log c)^{-1}+(2 z)^{-1}$. For $0 \neq n \in \mathbb{Z}$ the $n$th summand is $\pi(z \log c)^{-1} \exp (2 \pi i n \log z / \log c)\left(\sin \left(2 \pi^{2} i n / \log c\right)\right)^{-1}$. From this the asymptotic formula follows. The $n$th summand without the factor $\pi(z \log c)^{-1}$ is $\ll \exp (2 \pi|n|(\pi-\varepsilon) / \log c) \exp \left(-2 \pi^{2}|n| / \log c\right)$. Finally, assume $n_{0} \in \mathbb{N}$, $|z|=(1+c) c^{n_{0}} / 2$. For $n \geq n_{0}+1$ we have

$$
\left|z+c^{n}\right| \geq c^{n}-|z| \geq c^{n}-\frac{1+c}{2} c^{n-1}=\frac{c-1}{2 c} c^{n} \gg_{c} c^{n} .
$$

For $0 \leq n \leq n_{0}$

$$
\left|z+c^{n}\right| \geq|z|-c^{n} \geq|z|-c^{n_{0}}=|z|-\frac{2|z|}{c+1}=\frac{c-1}{c+1}|z| \gg_{c}|z| .
$$

From this it follows that

$$
\begin{aligned}
|G(z)| & \ll \sum_{0 \leq n \leq n_{0}}|z|^{-1}+\sum_{n \geq n_{0}+1} c^{-n} \\
& \ll_{c}\left(n_{0}+1\right)|z|^{-1}+c^{-n_{0}} \ll_{c}|z|^{-1} \log (|z|+2) .
\end{aligned}
$$

3. Proof of Theorem 1.3. Choose $\Theta<\Theta^{\prime}<\pi / 2$ with $\varrho_{0}:=\varrho(P)+$ $d(P) \Theta^{\prime}<\pi / 2$ and $0<a<\min _{\varrho, n} \Re \lambda_{\varrho n}$. Let $\gamma_{ \pm 1}(R)$ respectively $\gamma_{ \pm 1}$ be the paths which are parameterised by $z=r e^{ \pm i \Theta^{\prime}}, r \in\left[a / \cos \Theta^{\prime}, R\right]$ respectively $r \in\left[a / \cos \Theta^{\prime}, \infty\right)$, and $\gamma_{0}$ the path from $a e^{i \Theta^{\prime}} / \cos \Theta^{\prime}$ to $a e^{-i \Theta^{\prime}} / \cos \Theta^{\prime}$. Choose $p \in \mathbb{N}_{0}$ so that $S_{\varrho}(w), 1 \leq \varrho \leq r$, converge absolutely for $\Re w \geq p+1$.

Let $b \in \mathbb{R}^{-} \backslash \mathbb{Z}$ so that none of the $S_{\varrho}(w), 1 \leq \varrho \leq r$, has poles on $\Re w=b$. From Theorem 2.3 it follows that for $z \in \operatorname{Trace}\left(\gamma_{1}\right) \cup \operatorname{Trace}\left(\gamma_{-1}\right)$, $1 \leq \varrho \leq r$

$$
M S_{\varrho}(-z)=\sum_{\substack{1 \leq l \leq L_{\varrho} \\ 0 \leq \nu \leq a_{\varrho l}-1}} c_{\varrho l \nu}(-z)^{w_{\varrho l}-1} \log ^{\nu}(-z)+R_{\varrho}(z)
$$

with $\left|R_{\varrho}(z)\right| \ll_{b, \Theta^{\prime}}|z|^{b-1}$. The numbers $w_{\varrho l}, 1 \leq l \leq L_{\varrho}$, are the poles of $(\sin \pi w)^{-1} S_{\varrho}(w)$ in the vertical strip $B(b, p+1)$ and $a_{\varrho l}$ is the order of the pole $w_{\varrho l}$. The asymptotics (3.1) hold trivially also for $z \in \operatorname{Trace}\left(\gamma_{0}\right)$. Then $R_{\varrho}(z)$ is continuous on $\operatorname{Trace}\left(\gamma_{1}\right) \cup \operatorname{Trace}\left(\gamma_{0}\right) \cup \operatorname{Trace}\left(\gamma_{-1}\right)$ with the exception of the point $a$ where $R_{\varrho}(z)$ may have a discontinuity. As a special case it 
follows that

$$
\left|M S_{\varrho}(-z)\right| \ll|z|^{p} \log ^{K}|z| \quad \text { for } z \in \operatorname{Trace}\left(\gamma_{1}\right) \cup \operatorname{Trace}\left(\gamma_{-1}\right)
$$

with some $K>0$. Let $g$ be holomorphic on an open neighbourhood of

$$
S_{\Theta^{\prime}, a}:=\left\{w \in \mathbb{C}|\Re w \geq a,| \arg w \mid \leq \Theta^{\prime}\right\}
$$

and assume the estimate $|g(z)| \ll|z|^{-p-\alpha-1-\delta}$ on $S_{\Theta^{\prime}, a}$ with $\delta>0$. Choose $\left(R_{m}\right)_{m \geq 1}$ according to Lemma 2.4 and let $\mathcal{K}_{m}$ be the positively oriented arc of the circle around 0 with radius $R_{m}$ which lies inside $S_{\Theta^{\prime}}$. The residue theorem gives

$$
\frac{1}{2 \pi i} \int_{-\gamma_{1}\left(R_{m}\right)+\gamma_{0}+\gamma_{-1}\left(R_{m}\right)+\mathcal{K}_{m}} g(z) M S_{\varrho}(-z) d z=\sum_{n:\left|\lambda_{\varrho n}\right|<R_{m}}-a_{n}^{(\varrho)} g\left(\lambda_{\varrho n}\right) .
$$

The integral along $\mathcal{K}_{m}$ is $\ll R_{m}^{1-p-\alpha-1-\delta+p+\alpha}=R_{m}^{-\delta}$. Letting $m \rightarrow \infty$ gives

$$
\sum_{n \geq 1}-a_{n}^{(\varrho)} g\left(\lambda_{\varrho n}\right)=\frac{1}{2 \pi i} \int_{-\gamma_{1}+\gamma_{0}+\gamma_{-1}} g(z) M S_{\varrho}(-z) d z .
$$

The integral is absolutely convergent because of (3.2). For $w \in \mathbb{C}$ with $\Re w<p$, and $\nu \in \mathbb{N}_{0}$, Cauchy's theorem gives

$$
\begin{aligned}
& \int_{-\gamma_{1}+\gamma_{0}+\gamma_{-1}} g(z)(-z)^{w} \log ^{\nu}(-z) d z \\
& =\int_{\infty}^{a} g(t) e^{w(\log t-i \pi)}(\log t-i \pi)^{\nu} d t \\
& \quad+\int_{a}^{\infty} g(t) e^{w(\log t+i \pi)}(\log t+i \pi)^{\nu} d t \\
& =\int_{a}^{\infty} g(t) t^{w}\left(e^{i \pi w}(\log t+i \pi)^{\nu}-e^{-i \pi w}(\log t-i \pi)^{\nu}\right) d t \\
& =\sum_{\mu=0}^{\nu}\left(\begin{array}{l}
\nu \\
\mu
\end{array}\right)(i \pi)^{\nu-\mu} \int_{a}^{\infty} g(t) t^{w} \log ^{\mu} t d t\left(e^{i \pi w}-e^{-i \pi w}(-1)^{\nu-\mu}\right) .
\end{aligned}
$$

Let $\sigma_{\mathrm{a}}^{*}>0$ be the abscissa of absolute convergence of the Dirichlet series $\sum_{\underline{n} \in \mathbb{N}^{r}} P(\underline{n})^{-s}$. According to [9], Lemmas 3.2 and 4.2, the estimate

$$
|P(\underline{z})| \gg\left|z_{1} \ldots z_{r}\right|^{1 / \sigma_{\mathrm{a}}^{*}}
$$

holds for $\underline{z} \in S_{\Theta^{\prime}, a}^{r}$. It follows that $\sigma_{\mathrm{a}}(S) \leq \sigma_{\mathrm{a}}^{*}(p+1)$. For $\Re s>\sigma_{\mathrm{a}}^{*}(p+\alpha+1)$ it follows that with $\delta(s)>0$,

$$
\left|P(\underline{z})^{-s}\right| \ll_{s} e^{\varrho_{0}|\Im s|}\left|z_{1} \ldots z_{r}\right|^{-\Re s / \sigma_{\mathrm{a}}^{*}} \ll_{s}\left|z_{1} \ldots z_{r}\right|^{-(p+\alpha+1+\delta(s))}
$$


for $\underline{z} \in S_{\Theta^{\prime}, a}^{r} \cdot r$-fold application of (3.3) gives

$$
(-1)^{r} S(s)=\frac{1}{(2 \pi i)^{r}} \int_{\left(-\gamma_{1}+\gamma_{0}+\gamma_{-1}\right)^{r}} P(\underline{z})^{-s} \prod_{\varrho=1}^{r} M S_{\varrho}\left(-z_{\varrho}\right) d \underline{z} .
$$

Here integrations and summations may be interchanged because of the choice of $p$ and (3.2). Substituting (3.1) into (3.6) shows that $S(s)$ is a linear combination of the parameter integrals

$$
\int_{\left(-\gamma_{1}+\gamma_{0}+\gamma_{-1}\right)^{r}} P(\underline{z})^{-s} \prod_{\varrho \in K_{1}}\left(-z_{\varrho}\right)^{w_{\varrho l_{\varrho}}-1} \log ^{\nu_{\varrho}}\left(-z_{\varrho}\right) \prod_{\varrho \in K_{2}} R_{\varrho}\left(z_{\varrho}\right) d \underline{z}
$$

where $K_{1}$ runs through all subsets of $\{1, \ldots, r\},\left(l_{\varrho}\right)_{\varrho \in K_{1}}$ runs through $\prod_{\varrho \in K_{1}}\left\{1, \ldots, L_{\varrho}\right\},\left(\nu_{\varrho}\right)_{\varrho \in K_{1}}$ runs through $\prod_{\varrho \in K_{1}}\left\{0, \ldots, a_{\varrho l_{\varrho}}-1\right\}$ and $K_{2}:=$ $\{1, \ldots, r\} \backslash K_{1}$. With (3.4) one sees that each of these integrals is a linear combination of the integrals

$$
\begin{aligned}
\int_{\left(-\gamma_{1}+\gamma_{0}+\gamma_{-1}\right)^{\left|K_{2}\right|}} d\left(z_{\varrho}\right)_{\varrho \in K_{2}} \prod_{\varrho \in K_{2}} R_{\varrho}\left(z_{\varrho}\right) & \int_{[a, \infty)^{\left|K_{1}\right|}} P\left(\left(z_{\varrho}\right)_{\varrho \in K_{2}},\left(t_{\varrho}\right)_{\varrho \in K_{1}}\right)^{-s} \\
\times & \prod_{\varrho \in K_{1}} t_{\varrho}^{w_{\varrho l} l^{-1}} \log ^{\mu_{\varrho}} t_{\varrho} d\left(t_{\varrho}\right)_{\varrho \in K_{1}}
\end{aligned}
$$

where for each $\varrho \in K_{1}$ the parameter $\mu_{\varrho}$ runs through $\left\{0, \ldots, \nu_{\varrho}\right\}$. If $w_{\varrho l_{\varrho}} \in$ $\mathbb{Z}$ then $\mu_{\varrho}$ only runs through $\left\{0, \ldots, \nu_{\varrho}-1\right\} . S(s)$ is therefore a linear combination of the integrals (3.7) where $K_{1}$ runs through all subsets of $\{1, \ldots, r\},\left(l_{\varrho}\right)_{\varrho \in K_{1}}$ runs through $\prod_{\varrho \in K_{1}}\left\{1, \ldots, L_{\varrho}\right\},\left(\mu_{\varrho}\right)_{\varrho \in K_{1}}$ runs through $\prod_{\varrho \in K_{1}}\left\{0, \ldots\right.$, polord $\left._{w_{\varrho l_{\varrho}}}\left(S_{\varrho}\right)-1\right\}$ and $K_{2}:=\{1, \ldots, r\} \backslash K_{1}$. If polord $w_{w_{\varrho} l_{\varrho}}\left(S_{\varrho}\right)$ $=0$ for a $\varrho \in K_{1}$ then the corresponding integral does not appear.

The absolute convergence of the integrals (3.7) can be checked using $\Re s>\sigma_{\mathrm{a}}^{*}(p+\alpha+1)$. In the following they will be written as linear combinations of other integrals by decomposing the sets of integration and by the transformation formula. Therefore the subsequent integrals are absolutely convergent.

Decomposing the paths $-\gamma_{1}+\gamma_{0}+\gamma_{-1}$ and parameterizing $\gamma_{ \pm 1}$ show that (3.7) is a linear combination of the integrals

$$
\begin{aligned}
& \int_{\gamma_{0}^{\left|K_{21}\right|}} d\left(z_{\varrho}\right)_{\varrho \in K_{21}} \prod_{\varrho \in K_{21}} R_{\varrho}\left(z_{\varrho}\right) \int_{[1, \infty)^{\left|K_{22}\right|+\left|K_{1}\right|}} P_{j}\left(\underline{z}^{\prime},\left(t_{\varrho}\right)_{\left.\varrho \in K_{1} \cup K_{22}\right)^{-s}}\right. \\
& \times \prod_{\varrho \in K_{1}} t_{\varrho}^{w_{\varrho l}-1} \log ^{\mu_{\varrho}}\left(a t_{\varrho}\right) \prod_{\varrho \in K_{22}} R_{\varrho}\left(t_{\varrho} \frac{a}{\cos \Theta^{\prime}} e^{i j_{\varrho} \Theta^{\prime}}\right) d\left(t_{\varrho}\right)_{\varrho \in K_{1} \cup K_{22}}
\end{aligned}
$$

with

$$
P_{\underline{j}}\left(\underline{Z}^{\prime},\left(T_{\varrho}\right)_{\varrho \in K_{1} \cup K_{22}}\right):=P\left(\left(Z_{\varrho}\right)_{\varrho \in K_{21}},\left(T_{\varrho} \frac{a}{\cos \Theta^{\prime}} e^{i j_{\varrho} \Theta^{\prime}}\right)_{\varrho \in K_{22}},\left(a T_{\varrho}\right)_{\varrho \in K_{1}}\right)
$$


where $\underline{z}^{\prime}=\left(z_{\varrho}\right)_{\varrho \in K_{21}}, K_{21}$ runs through all subsets of $K_{2}, K_{22}:=K_{2} \backslash K_{21}$ and $j=\left(j_{\varrho}\right)_{\varrho \in K_{22}}$ runs through $\{ \pm 1\}^{\left|K_{22}\right|}$. We have

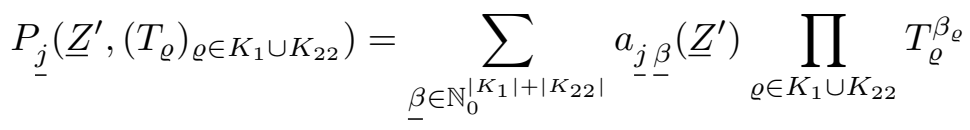

with

$$
a_{\underline{j} \underline{\beta}}\left(\underline{Z}^{\prime}\right)=\sum_{\underline{\beta}^{\prime} \in \mathbb{N}_{0}^{\left|K_{21}\right|}} c_{\left(\underline{\beta}, \underline{\beta}^{\prime}\right)} \prod_{\varrho \in K_{1}} a^{\beta_{\varrho}} \prod_{\varrho \in K_{22}}\left(\frac{a}{\cos \Theta^{\prime}} e^{i j_{\varrho} \Theta^{\prime}}\right)^{\beta_{\varrho}} \prod_{\varrho \in K_{21}} Z_{\varrho}^{\beta_{\varrho}^{\prime}} .
$$

From this it follows that $\Re a_{\underline{j}} \underline{z^{\prime}} \underline{z}^{\prime}>0$ for each $\underline{z}^{\prime} \in \gamma_{0}^{\left|K_{21}\right|}$ if there is a $\underline{\beta}^{\prime} \in \mathbb{N}_{0}^{\left|K_{21}\right|}$ with $c_{\left(\beta, \beta^{\prime}\right)} \neq 0$. Theorem 2.1 yields the existence of $\omega(\underline{\underline{\lambda}}) \in \Omega$, $\underline{\underline{\lambda}} \in \Lambda\left(K_{1}, K_{21}\right)$, which depend only upon $K_{1}, K_{21}$ with the properties: $\overline{\overline{(1}}, \infty)^{\left|K_{1}\right|+\left|K_{22}\right|}$ is up to sets of measure zero the disjoint union of the sets $\operatorname{Img} \omega(\underline{\underline{\lambda}})$ and for each $\underline{\underline{\lambda}} \in \Lambda\left(K_{1}, K_{21}\right)$,

$$
\begin{aligned}
Q_{\underline{j} \underline{\underline{\lambda}}}\left(\left(Z_{\varrho}\right)_{\varrho \in K_{21}},\left(T_{\varrho}\right)_{\varrho \in K_{1} \cup K_{22}}\right) & =P_{\underline{j} \omega(\underline{\underline{\lambda}})} \\
& =\sum_{\underline{\gamma} \leq \underline{\gamma}^{\prime}} b_{\underline{\gamma}}^{\underline{\underline{\lambda}}}\left(\underline{\underline{\lambda}}\left(\underline{Z}^{\prime}\right) \prod_{\varrho \in K_{1} \cup K_{22}} T_{\varrho}^{\gamma_{\varrho}} .\right.
\end{aligned}
$$

Here $b_{\underline{\gamma}}^{\underline{j}} \stackrel{\underline{\lambda}}{=}\left(\underline{Z}^{\prime}\right) \in \mathbb{C}\left[\underline{Z}^{\prime}\right]$ with $\Re b_{\underline{\gamma}}^{\underline{j}} \underline{\underline{\lambda}}\left(\underline{z}^{\prime}\right)>0$ if $\underline{z}^{\prime} \in \gamma_{0}^{\left|K_{21}\right|}$ and $b_{\underline{\gamma}}^{\underline{j}} \underline{\underline{\lambda}} \not \equiv 0 ; \underline{\gamma}^{\prime}=\underline{\gamma}^{\prime}(\underline{\underline{\lambda}})$ depends on $\underline{\underline{\lambda}}$. Furthermore, $b_{\underline{\gamma^{\prime}}}^{-\underline{\underline{\lambda}}} \not \equiv 0$.

Decomposing the region of integration in the innermost integral in (3.8) and applying the transformation formula show that (3.7) is a linear combination of integrals

$$
\int_{\gamma_{0}^{\left|K_{21}\right|}} d\left(z_{\varrho}\right)_{\varrho \in K_{21}} \prod_{\varrho \in K_{21}} R_{\varrho}\left(z_{\varrho}\right) \int_{(1, \infty)^{\left|K_{1}\right|+\left|K_{22}\right|}} d\left(\tau_{\varrho}\right)_{\varrho \in K_{1} \cup K_{22}}
$$

$$
\begin{aligned}
& \times Q_{\underline{j} \underline{\underline{\lambda}}}\left(\left(z_{\varrho}\right)_{\varrho \in K_{21}},\left(\tau_{\varrho}\right)_{\varrho \in K_{1} \cup K_{22}}\right)^{-s} \prod_{\varrho \in K_{22}} R_{\varrho}\left(\frac{a}{\cos \Theta^{\prime}} e^{i j_{\varrho} \Theta^{\prime}} \prod_{\mu \in K_{1} \cup K_{22}} \tau_{\mu}^{\lambda_{\mu \varrho}}\right) \\
& \times \prod_{\mu \in K_{1} \cup K_{22}} \tau_{\mu}^{\alpha_{\mu}} \prod_{\mu \in K_{1} \cup K_{22}} \log ^{\kappa_{\mu}} \tau_{\mu}
\end{aligned}
$$

where $K_{21}$ runs through all subsets of $K_{2}, K_{22}:=K_{2} \backslash K_{21}, j=\left(j_{\varrho}\right)_{\varrho \in K_{22}}$ runs through $\{ \pm 1\}^{\left|K_{22}\right|}, \underline{\underline{\lambda}}=\left(\lambda_{\varrho \mu}\right)$ runs through a finite set $\Lambda\left(K_{1}, K_{21}\right)$ of regular matrices $\in \mathbb{N}_{0}^{\left(\left|K_{1}\right| \overline{+}\left|K_{22}\right|\right)^{2}},\left(\kappa_{\varrho}\right)_{\varrho \in K_{1} \cup K_{22}}$ runs through all elements of $\mathbb{N}_{0}^{\left|K_{1}\right|+\left|K_{22}\right|}$ with $\sum_{\varrho \in K_{1} \cup K_{22}} \kappa_{\varrho} \leq \sum_{\varrho \in K_{1}} \mu_{\varrho}$ and $Q_{j \underline{\underline{\lambda}}} \in \mathbb{C}\left[\underline{Z}^{\prime}, \underline{T}\right]$ is of the 
form (3.9). Finally,

$$
\alpha_{\mu}:=\sum_{\varrho \in K_{1}} \lambda_{\mu \varrho}\left(w_{\varrho l_{\varrho}}-1\right)-1+\sum_{\varrho \in K_{1} \cup K_{22}} \lambda_{\mu \varrho}, \quad \mu \in K_{1} \cup K_{22} .
$$

From $\operatorname{deg}_{X_{\varrho}} P \geq 1$ for each $1 \leq \varrho \leq r$ it follows that

$$
\lim _{\substack{z \in S_{\Theta^{\prime}, a}^{r} \\|\underline{z}| \rightarrow \infty}}|P(\underline{z})|=\infty .
$$

Therefore,

$$
\lim _{\substack{\underline{\tau} \in(1, \infty)^{\left|K_{1}\right|+\left|K_{22}\right|} \\ \mid \underline{\underline{\tau} \mid \rightarrow \infty}}}\left|Q_{\underline{j} \underline{\underline{\lambda}}}\left((a)_{\varrho \in K_{21}}, \underline{\tau}\right)\right|=\infty
$$

and thus $\gamma_{\mu}^{\prime} \geq 1$ for each $\mu \in K_{1} \cup K_{22}$.

Define $U=U(\underline{\underline{\lambda}}):=\left\{\mu \in K_{1} \cup K_{22} \mid \lambda_{\mu \varrho} \geq 1\right.$ for a $\left.\varrho \in K_{22}\right\}$ and $V=V(\underline{\underline{\lambda}}):=\left(K_{1} \cup K_{22}\right) \backslash U$. After substitution of $\tau_{\mu}^{-1}$ for $\tau_{\mu}$ the integral (3.10) is

$$
\begin{aligned}
& \pm \int_{\gamma_{0}^{\left|K_{21}\right|}} d\left(z_{\varrho}\right)_{\varrho \in K_{21}} \prod_{\varrho \in K_{21}} R_{\varrho}\left(z_{\varrho}\right) \int_{(0,1)^{|U|}} d\left(\tau_{\mu}\right)_{\mu \in U} R\left(\left(\tau_{\mu}\right)_{\mu \in U}\right) \\
& \times \prod_{\mu \in U} \tau_{\mu}^{-\alpha_{\mu}-2} \log ^{\kappa_{\mu}} \tau_{\mu} \int_{(0,1)^{|V|}} Q_{\underline{j}}\left(\underline{\underline{\lambda}}\left(\left(z_{\varrho}\right)_{\varrho \in K_{21}},\left(\tau_{\mu}^{-1}\right)_{\mu \in K_{1} \cup K_{22}}\right)^{-s}\right. \\
& \quad \times \prod_{\mu \in V} \tau_{\mu}^{-\alpha_{\mu}-2} \log ^{\kappa_{\mu}} \tau_{\mu} d\left(\tau_{\mu}\right)_{\mu \in V}
\end{aligned}
$$

with

$$
R\left(\left(\tau_{\mu}\right)_{\mu \in U}\right)=R_{\underline{j, \underline{\lambda}}}:=\prod_{\varrho \in K_{22}} R_{\varrho}\left(\frac{a}{\cos \Theta^{\prime}} e^{i j_{\varrho} \Theta^{\prime}} \prod_{\mu \in U} \tau_{\mu}^{-\lambda_{\mu \varrho}}\right) .
$$

Therefore

$$
\begin{aligned}
R\left(\left(\tau_{\mu}\right)_{\mu \in U}\right) & \ll \prod_{\varrho \in K_{22}}\left(\prod_{\mu \in U} \tau_{\mu}^{-\lambda_{\mu \varrho}}\right)^{b-1}=\prod_{\mu \in U} \tau_{\mu}^{(1-b) \sum_{\varrho \in K_{22}} \lambda_{\mu \varrho}} \\
& \ll \prod_{\mu \in U} \tau_{\mu}^{1-b}
\end{aligned}
$$

For $\underline{z}^{\prime}=\left(z_{\varrho}\right)_{\varrho \in K_{21}} \in \gamma_{0}^{\left|K_{21}\right|}$ and $\underline{\tau}=\left(\tau_{\mu}\right)_{\mu \in K_{1} \cup K_{22}} \in[0,1]^{\left|K_{1}\right|+\left|K_{22}\right|}$ we have $\Re\left(\sum_{\underline{\gamma} \leq \underline{\gamma^{\prime}}} b_{\underline{\gamma}}^{\underline{j}}=\underline{\underline{\lambda}}\left(\underline{z}^{\prime}\right) \underline{\tau}^{\gamma^{\prime}-\underline{\gamma}}\right)>0$. With a continuity argument it follows from the compactness of $\gamma_{0}^{\left|K_{21}\right|} \times[0,1]^{\left|K_{1}\right|+\left|K_{22}\right|} \subseteq \mathbb{C}^{r}$ that there are $\varepsilon, c>0$ with the property: For each $\underline{z}^{\prime} \in \gamma_{0}^{\left|K_{21}\right|},\left(\tau_{\mu}\right)_{\mu \in U} \in[0,1]^{|U|}$ and $\left(v_{\mu}\right)_{\mu \in V} \in\{w \in \mathbb{C} \mid$ 
$-\varepsilon \leq \Re w \leq 1+\varepsilon,|\Im w| \leq \varepsilon\}^{|V|}$,

$$
\Re\left(\sum_{\underline{\gamma} \leq \underline{\gamma}^{\prime}} b_{\underline{\gamma}}^{\underline{\underline{\lambda}}} \underline{\underline{\lambda}}\left(\underline{z}^{\prime}\right) \prod_{\mu \in U} \tau_{\mu}^{\gamma_{\mu}^{\prime}-\gamma_{\mu}} \prod_{\mu \in V} v_{\mu}^{\gamma_{\mu}^{\prime}-\gamma_{\mu}}\right) \geq c>0 .
$$

It follows from Lemma 2.5 for $\Re s>\max \left\{\sigma_{\mathrm{a}}^{*}(p+\alpha+1),\left(\Re \alpha_{\mu}+1\right) / \gamma_{\mu}^{\prime} \mid \mu \in\right.$ $\left.K_{1} \cup K_{22}\right\}, s \gamma_{\mu}^{\prime}-\alpha_{\mu} \notin \mathbb{Z}$ for each $\mu \in V$, that (3.10) has the representation

$$
\begin{aligned}
\pm \sum_{\substack{0 \leq \lambda_{\mu} \leq \kappa_{\mu} \\
\mu \in V}} & \prod_{\mu \in V} \frac{P_{\kappa_{\mu} \lambda_{\mu}}\left(e\left(s \gamma_{\mu}^{\prime}-\alpha_{\mu}-2\right)\right)}{\left(e\left(s \gamma_{\mu}^{\prime}-\alpha_{\mu}-2\right)-1\right)^{\kappa_{\mu}+1-\lambda_{\mu}}} \\
& \times \int_{\gamma_{0}^{\left|K_{21}\right|}} d\left(z_{\varrho}\right)_{\varrho \in K_{21}} \prod_{\varrho \in K_{21}} R_{\varrho}\left(z_{\varrho}\right) \\
& \times \int_{(0,1)^{|U|}} d\left(\tau_{\mu}\right)_{\mu \in U} R\left(\left(\tau_{\mu}\right)_{\mu \in U}\right) \prod_{\mu \in U} \tau_{\mu}^{s \gamma_{\mu}^{\prime}-\alpha_{\mu}-2} \log ^{\kappa_{\mu}} \tau_{\mu} \\
& \times \int_{I_{\varepsilon}^{|V|}}\left(\sum_{\underline{\gamma} \leq \underline{\gamma}^{\prime}} b^{\underline{\underline{\gamma}}} \underline{\underline{\lambda}}\left(\left(z_{\varrho}\right)_{\varrho \in K_{21}}\right) \prod_{\mu \in U} \tau_{\mu}^{\gamma_{\mu}^{\prime}-\gamma_{\mu}} \prod_{\mu \in V} v_{\mu}^{\gamma_{\mu}^{\prime}-\gamma_{\mu}}\right)^{-s} \\
& \times \prod_{\mu \in V} v_{\mu}^{s \gamma_{\mu}^{\prime}-\alpha_{\mu}-2} \log ^{\lambda_{\mu}} v_{\mu} d\left(v_{\mu}\right)_{\mu \in V} .
\end{aligned}
$$

In fact, it is possible to deduce from (3.5) and (3.9) that

$$
\sigma_{\mathrm{a}}^{*} \gamma_{\mu}^{\prime} \geq \sum_{\varrho \in K_{1} \cup K_{22}} \lambda_{\mu \varrho}
$$

for $\mu \in K_{1} \cup K_{22}$. Therefore $\sigma_{\mathrm{a}}^{*}(p+\alpha+1)>\left(\Re \alpha_{\mu}+1\right) / \gamma_{\mu}^{\prime}$ for each $\mu \in$ $K_{1} \cup K_{22}$ but this fact will not be used in the sequel.

From (3.11) it follows that each of the integrals in (3.13) defines a holomorphic function with respect to $s$ as long as $\Re\left(s \gamma_{\mu}^{\prime}-\alpha_{\mu}-2\right)+1-b>-1$ for each $\mu \in U$. From

$$
\max _{\mu \in U} \gamma_{\mu}^{\prime-1}\left(b+\Re \alpha_{\mu}\right) \leq \max _{\mu \in U} \gamma_{\mu}^{\prime-1}\left(b+\sum_{\varrho \in K_{1}} \lambda_{\mu \varrho} p-1+\sum_{\varrho \in K_{1} \cup K_{22}} \lambda_{\mu \varrho}\right)
$$

the existence of constants $c_{1}, c_{2}>0$ can be deduced so that (3.10) can be continued meromorphically to $\Re s>c_{1} b+c_{2}$. For $\mu \in V$ define $L_{\overline{\bar{\mu}}}^{\lambda}\left(\left(Y_{\varrho}\right)_{\varrho \in K_{1}}\right)$ $:=\sum_{\varrho \in K_{1}} \lambda_{\mu \varrho} Y_{\varrho}$. Then the poles of (3.10) which are contained in $\Re s>$ $c_{1} b+c_{2}$ lie in the set

$$
\bigcup_{\mu \in V} \gamma_{\mu}^{\prime-1}\left(L_{\bar{\mu}}^{\underline{\lambda}}\left(\left(w_{\varrho l_{\varrho}}\right)_{\varrho \in K_{1}}\right)+\mathbb{Z}\right)
$$


The order of a pole $w_{0}$ with $\Re w_{0}>c_{1} b+c_{2}$ is

$$
\leq \sum_{\mu \in V}\left(\kappa_{\mu}+1\right) \leq \sum_{\varrho \in K_{1}} \mu_{\varrho}+|V| \leq \sum_{\varrho \in K_{1}}\left(\operatorname{polord}_{w_{\varrho l_{\varrho}}}\left(S_{\varrho}\right)-1\right)+r .
$$

$w_{0}$ can be a pole of (3.10) only if there are $\mu \in V$ and $a \in \mathbb{Z}$ with $w_{0}=$ $\gamma_{\mu}^{\prime-1}\left(a+L_{\mu}^{\underline{\lambda}}\left(\left(w_{\varrho} l_{\varrho}\right)_{\varrho \in K_{1}}\right)\right)$. Let

$$
\begin{aligned}
& N:=\operatorname{lcm}\left\{\gamma_{\mu}^{\prime}(\underline{\underline{\lambda}}) \mid \mu \in V(\underline{\underline{\lambda}}), \underline{\underline{\lambda}} \in \Lambda\left(K_{1}, K_{21}\right),\right. K_{1} \subseteq\{1, \ldots, r\}, \\
&\left.K_{21} \subseteq\{1, \ldots, r\} \backslash K_{1}\right\}, \\
& \mathcal{L}\left(K_{1}, K_{21}, \underline{\underline{\lambda}}\right):=\left\{N \gamma_{\mu}^{\prime}(\underline{\underline{\underline{\lambda}}})^{-1} L_{\underline{\mu}}^{\underline{\underline{\lambda}}} \mid \mu \in V(\underline{\underline{\lambda}})\right\} .
\end{aligned}
$$

Then (3.7) can be continued meromorphically to $\Re s>c_{1} b+c_{2}$, the poles lie in

$$
\begin{aligned}
\left\{N^{-1}\left(L\left(\left(w_{\varrho l_{\varrho}}\right)_{\varrho \in K_{1}}\right)+a\right) \mid a \in \mathbb{Z}, L \in \mathcal{L}\left(K_{1}, K_{21}, \underline{\underline{\lambda}}\right),\right. & \\
& \left.\quad \underline{\underline{\lambda}} \in \Lambda\left(K_{1}, K_{21}\right), K_{21} \subseteq K_{2}\right\}
\end{aligned}
$$

and have an order $\leq \sum_{\varrho \in K_{1}}\left(\operatorname{polord}_{w_{\varrho l_{\varrho}}}\left(S_{\varrho}\right)-1\right)+r$. Taking all together one sees that $S(s)$ can be continued meromorphically to $\Re s>c_{1} b+c_{2}$ and that the poles lie in

$$
\begin{aligned}
& \left\{N^{-1}\left(L\left(\left(w_{\varrho l_{\varrho}}\right)_{\varrho \in K_{1}}\right)+a\right) \mid a \in \mathbb{Z}, L \in \mathcal{L}\left(K_{1}, K_{21}, \underline{\underline{\lambda}}\right), \underline{\underline{\lambda}} \in \Lambda\left(K_{1}, K_{21}\right),\right. \\
& \left.K_{21} \subseteq\{1, \ldots, r\} \backslash K_{1},\left(l_{\varrho}\right)_{\varrho \in K_{1}} \subseteq \prod_{\varrho \in K_{1}}\left\{1, \ldots, L_{\varrho}\right\}, K_{1} \subseteq\{1, \ldots, r\}\right\} .
\end{aligned}
$$

As $b \in \mathbb{R}^{-} \backslash \mathbb{Z}$ can be chosen arbitrarily with the restriction that none of the $S_{\varrho}(w)$ has a pole on $\Re w=b$ one can continue $S(s)$ meromorphically to $\mathbb{C}$ and it has only a finite number of poles in each vertical strip of finite width. If $w_{0} \in \mathbb{C}$ is a pole of $S(s)$ then $\Re w_{0} \leq \sigma_{\mathrm{a}}(S)$. Choose $b$ such that $b<c_{1}^{-1}\left(\Re w_{0}-\left|\sigma_{\mathrm{a}}(S)\right|-c_{2}\right)<b+1$. Then $c_{1} b+c_{2}<\Re w_{0}$ and $b>c_{1}^{-1} \Re w_{0}-c_{3}$ with a constant $c_{3}>0$. There are necessarily $K_{1},\left(l_{\varrho}\right)_{\varrho \in K_{1}}, K_{21}, \underline{\underline{\lambda}}, L, a$ with $w_{0}=N^{-1}\left(L\left(\left(w_{\varrho l_{\varrho}}\right)_{\varrho \in K_{1}}\right)+a\right)$ where $w_{\varrho l_{\varrho}}$ is a pole of $S_{\varrho}$ in $B(\overline{\bar{b}}, p+1)$ for each $\varrho \in K_{1}$. Therefore

$$
\begin{aligned}
\Re\left(a+L\left(\left(w_{\varrho l_{\varrho}}\right)_{\varrho \in K_{1}}\right)\right) & =N \Re w_{0} \leq N c_{1}\left(b+c_{3}\right) \\
& \leq N c_{1} c_{3}+N c_{1} \min \left\{\Re w_{\varrho l_{\varrho}} \mid \varrho \in K_{1}\right\} .
\end{aligned}
$$

This gives (1.3) and (1.4). The inequality in (1.4) makes the set finite and therefore the maximum is well defined. The inequality in (1.3) ensures that for the poles of $S(s)$ in a finite strip, only a finite number of $w_{\varrho}$ need to be taken into consideration.

4. Proof of Theorem 1.4. DIR 1 and DIR3 are valid for $S_{\varrho}$ because of the general assumption $\lambda_{\varrho n} \in S_{\Theta}$. According to Corollary 1.10 of [3] the 
functions $\Gamma S_{\varrho}, 1 \leq \varrho \leq r$, can be continued meromorphically to $\mathbb{C}$ where they have poles at the points

$$
-p, p \in \mathcal{P}_{\varrho} \quad \text { of order } \quad \operatorname{deg} B_{p}^{(\varrho)}+1 .
$$

Together with the other assumptions it follows that Theorems 1.3 and 1.2 can be applied. Therefore $S \in \mathcal{K}$ and the poles of $S$ lie in a set of the form (1.3). Obviously $S(s)$ fulfils DIR1, DIR2(a) and DIR3. As each $S_{\varrho}$ fulfils DIR2(b) there is $\alpha>0$ with

$$
\sum_{n \geq 1}\left|\lambda_{\varrho n}\right|^{-\alpha}<\infty \quad \text { for each } 1 \leq \varrho \leq r .
$$

From (3.5) it follows that

$$
\sum_{n_{1}, \ldots, n_{r} \geq 1}\left|P\left(\lambda_{1 n_{1}}, \ldots, \lambda_{r n_{r}}\right)\right|^{-\sigma_{\mathrm{a}}^{*} \alpha} \ll \sum_{n_{1}, \ldots, n_{r} \geq 1}\left|\lambda_{1 n_{1}} \ldots \lambda_{r n_{r}}\right|^{-\sigma_{\mathrm{a}}^{*} \alpha / \sigma_{\mathrm{a}}^{*}}<\infty
$$

and therefore $S(s)$ fulfils DIR2(b). As $S \in \mathcal{K}$ the function $S(s)$ lies in $\operatorname{Dom}(V)$ in the notation of [3], p. 78. According to Theorem 7.5 of [3] the theta series $\Theta(t)$ fulfils AS1-AS3. According to Theorem 7.4 of [3], $\mathcal{P}=\left\{-w_{0} \mid w_{0}\right.$ a pole of $\left.\Gamma(s) S(s)\right\}$ and therefore $\mathcal{P}$ is contained in a set of the form (1.5). If $p \in \mathcal{P}$ then it follows from (9), p. 80 of [3] that

$$
\operatorname{deg} B_{p}=\text { polord }_{-p}(\Gamma(s) S(s))-1=\text { polord }_{-p} S(s)+\varepsilon(p)-1 .
$$

From (4.1) and (1.4) the inequality (1.6) follows.

5. Proof of Theorem 1.5. According to Lemma 2.7 the Dirichlet series

$$
S(s):=\sum_{n_{1}, \ldots, n_{r} \geq 1} \frac{\Lambda\left(n_{1}\right) \ldots \Lambda\left(n_{r}\right)}{P\left(n_{1}, \ldots, n_{r}\right)^{s}}
$$

has the abscissa of (absolute) convergence

$$
\sigma_{\mathrm{a}}=\sigma_{\mathrm{a}}(S)=\min \left\{\sigma>0 \mid P(\underline{x})^{\sigma} \gg_{\sigma} x_{1} \ldots x_{r} \text { for } \underline{x} \in(1, \infty)^{r}\right\} .
$$

Choose $\Theta^{\prime}>0$ with $\varrho_{0}:=d(P) \Theta^{\prime}<\pi / 2$. Assume $\Re s>\sigma_{\mathrm{a}}$. According to Lemma 4.2 of [9] for each $\underline{z} \in S_{\Theta^{\prime}}^{r}$ with $\left|z_{1}\right|, \ldots,\left|z_{r}\right| \geq 1$,

$$
\left|P(\underline{z})^{s}\right| \geq|P(\underline{z})|^{\Re s} e^{-|\Im s| \varrho_{0}} \gg_{s}\left|z_{1} \ldots z_{r}\right|^{\Re s / \sigma_{\mathrm{a}}} .
$$

From this it follows by Lemma 2.6 that

$$
(-1)^{r} S(s)=\frac{1}{2 \pi i} \int_{\left(-\gamma_{1}+\gamma_{0}+\gamma_{-1}\right)^{r}} P(\underline{z})^{-s} \prod_{\varrho=1}^{r} G\left(-z_{\varrho}\right) d \underline{z}
$$

where $a=1 / 2$ is used in the definition of $\gamma_{0}$. The integral is absolutely convergent for $\Re s>\sigma_{\mathrm{a}}$. The further transformations are as in the proof of 
Theorem 1.3 where (2.5) takes the role of (3.1). From this it follows that $S(s)$ is a linear combination of integrals of the form

$$
\begin{gathered}
\int_{\gamma_{0}^{\left|K_{21}\right|}} d\left(z_{\varrho}\right)_{\varrho \in K_{21}} \prod_{\varrho \in K_{21}} R\left(-z_{\varrho}\right) \int_{(0,1)^{|U|}} d\left(\tau_{\mu}\right)_{\mu \in U} \widetilde{R}\left(\left(\tau_{\mu}\right)_{\mu \in U}\right) \prod_{\mu \in U} \tau_{\mu}^{-\alpha_{\mu}-2} \\
\times \int_{(0,1)^{|V|}} Q\left(\left(z_{\varrho}\right)_{\varrho \in K_{21}},\left(\tau_{\mu}^{-1}\right)_{\mu \in U \cup V}\right)^{-s} \prod_{\mu \in V} \tau_{\mu}^{-\alpha_{\mu}-2} d\left(\tau_{\mu}\right)_{\mu \in V} .
\end{gathered}
$$

Here $U \dot{\cup} V \dot{\cup} K_{21}=\{1, \ldots, r\}, \alpha_{\mu} \in \mathbb{N}_{0}$ for $\mu \in U \cup V, Q \in \mathbb{C}\left[\underline{Z}^{\prime}, \underline{T}\right]$ has the form (3.9) and

$$
\begin{aligned}
\widetilde{R}\left(\left(\tau_{\mu}\right)_{\mu \in U}\right) & =\prod_{\varrho \in K_{22}} R\left(\frac{-1}{2 \cos \Theta^{\prime}} e^{ \pm i \Theta^{\prime}} \prod_{\mu \in U} \tau_{\mu}^{-\lambda_{\mu \varrho}}\right) \\
& \ll \prod_{\mu \in U} \exp \left(-c^{\prime} \frac{\log \left(\tau_{\mu}^{-1}+2\right)}{\log \log \left(\tau_{\mu}^{-1}+2\right)}\right)
\end{aligned}
$$

for $\left(\tau_{\mu}\right)_{\mu \in U} \in(0,1)^{|U|}$ with $c^{\prime}>0$. For $\left(z_{\varrho}\right)_{\varrho \in K_{21}} \in \gamma_{0}^{\left|K_{21}\right|}$ and $\left(t_{\varrho}\right)_{\varrho \in U \cup V} \in$ $(1, \infty)^{|U|+|V|}$ it follows from (5.3) that

$$
\begin{aligned}
& \left|Q\left(\left(z_{\varrho}\right)_{\varrho \in K_{21}},\left(t_{\mu}\right)_{\mu \in U \cup V}\right)^{-s}\right| \\
& \quad \ll_{s}\left(\prod_{\varrho \in U \cup V} \prod_{\mu \in U \cup V} t_{\mu}^{\lambda_{\mu \varrho}}\right)^{-\Re s / \sigma_{\mathrm{a}}}=\left(\prod_{\mu \in U \cup V} t_{\mu}^{\alpha_{\mu}+1}\right)^{-\Re s / \sigma_{\mathrm{a}}},
\end{aligned}
$$

and consequently (5.5) is absolutely convergent for $\Re s>\sigma_{\mathrm{a}}$. From (3.9) and (5.7) it follows that $\gamma_{\mu}^{\prime} \geq\left(\alpha_{\mu}+1\right) / \sigma_{\mathrm{a}}$ for each $\mu \in U \cup V$. If one chooses a suitable $\varepsilon>0$ then it follows by Lemma 2.5 and (3.12) that for each $\Re s>\sigma_{\mathrm{a}}$ with $s \gamma_{\mu}^{\prime} \notin \mathbb{Z}$ for $\mu \in V,(5.5)$ can be expressed in the form

$$
\begin{aligned}
& \prod_{\mu \in V}\left(e\left(s \gamma_{\mu}^{\prime}\right)-1\right)^{-1} \int_{\gamma_{0}^{\left|K_{21}\right|}} d\left(z_{\varrho}\right)_{\varrho \in K_{21}} \prod_{\varrho \in K_{21}} R\left(-z_{\varrho}\right) \\
& \times \int_{(0,1)^{|U|}} d\left(\tau_{\mu}\right)_{\mu \in U} \widetilde{R}\left(\left(\tau_{\mu}\right)_{\mu \in U}\right) \prod_{\mu \in U} \tau_{\mu}^{s \gamma_{\mu}^{\prime}-\alpha_{\mu}-2} \\
& \times \int_{I_{\varepsilon}^{|V|}}\left(\sum_{\underline{\gamma} \leq \underline{\gamma}^{\prime}} b_{\underline{\gamma}}\left(\left(z_{\varrho}\right)_{\varrho \in K_{21}}\right) \prod_{\mu \in U} \tau_{\mu}^{\gamma_{\mu}^{\prime}-\gamma_{\mu}} \prod_{\mu \in V} z_{\mu}^{\gamma_{\mu}^{\prime}-\gamma_{\mu}}\right)^{-s} \prod_{\mu \in V} z_{\mu}^{s \gamma_{\mu}^{\prime}-\alpha_{\mu}-2} d\left(z_{\mu}\right)_{\mu \in V} .
\end{aligned}
$$

The triple integral $I(s)$ which occurs in this expression is holomorphic in $\Re s>\sigma_{\mathrm{a}}$. For each $m \in \mathbb{N}_{0}$ the $m$ th derivative $I^{(m)}(s)$ can be represented as 
a linear combination of integrals of the form

$$
\begin{aligned}
& \int_{\gamma_{0}^{\left|K_{21}\right|}} d\left(z_{\varrho}\right)_{\varrho \in K_{21}} \prod_{\varrho \in K_{21}} R\left(-z_{\varrho}\right) \\
& \quad \times \int_{(0,1)^{|U|}} d\left(\tau_{\mu}\right)_{\mu \in U} \widetilde{R}\left(\left(\tau_{\mu}\right)_{\mu \in U}\right) \prod_{\mu \in U} \tau_{\mu}^{s \gamma_{\mu}^{\prime}-\alpha_{\mu}-2} \log ^{\beta_{\mu}} \tau_{\mu} \\
& \times \int_{I_{\varepsilon}^{|V|}} d\left(z_{\mu}\right)_{\mu \in V}\left(\sum_{\underline{\gamma} \leq \underline{\gamma}^{\prime}} \underline{b}_{\gamma}\left(\left(z_{\varrho}\right)_{\varrho \in K_{21}}\right) \prod_{\mu \in U} \tau_{\mu}^{\gamma_{\mu}^{\prime}-\gamma_{\mu}} \prod_{\mu \in V} z_{\mu}^{\gamma_{\mu}^{\prime}-\gamma_{\mu}}\right)^{-s} \\
& \quad \times \log ^{\beta}\left(\sum_{\underline{\gamma} \leq \underline{\gamma}^{\prime}} \underline{\underline{\gamma}}_{\underline{\gamma}}\left(\left(z_{\varrho}\right)_{\varrho \in K_{21}}\right) \prod_{\mu \in U} \tau_{\mu}^{\gamma_{\mu}^{\prime}-\gamma_{\mu}} \prod_{\mu \in V} z_{\mu}^{\gamma_{\mu}^{\prime}-\gamma_{\mu}}\right) \\
& \quad \times \prod_{\mu \in V} z_{\mu}^{s \gamma_{\mu}^{\prime}-\alpha_{\mu}-2} \log ^{\beta_{\mu}} z_{\mu}
\end{aligned}
$$

with $\beta_{\mu}, \beta \in \mathbb{N}_{0}$. From (5.6) it can be seen that these integrals are uniformly convergent on each compact subset of $\Re s \geq \sigma_{\mathrm{a}}$. Therefore $I^{(m)}(s)$ can be continued to a continuous function on $\Re s \geq \sigma_{\mathrm{a}}$.

For $z_{\varrho} \in \gamma_{0}, \varrho \in K_{21}, \tau_{\mu} \in(0,1), \mu \in U, z_{\mu} \in I_{\varepsilon}, \mu \in V$,

$$
\left|\sum_{\underline{\gamma} \leq \underline{\gamma}^{\prime}} b_{\underline{\gamma}}\left(\left(z_{\varrho}\right)_{\varrho \in K_{21}}\right) \prod_{\mu \in U} \tau_{\mu}^{\gamma_{\mu}^{\prime}-\gamma_{\mu}} \prod_{\mu \in V} z_{\mu}^{\gamma_{\mu}^{\prime}-\gamma_{\mu}}\right| \asymp 1 .
$$

Consequently, (5.8) can be estimated for $\sigma_{\mathrm{a}} \leq \Re s \leq \sigma_{\mathrm{a}}+1$ by $O(\exp (|\Im s|(\pi / 2$ $\left.\left.\left.+2 \pi \sum_{\mu \in V} \gamma_{\mu}^{\prime}\right)\right)\right)$ and so $\left|I^{(m)}(s)\right| \ll_{m} \exp (c|\Im s|)$ for $\sigma_{\mathrm{a}} \leq \Re s \leq \sigma_{\mathrm{a}}+1$, $m \in \mathbb{N}_{0}$ with a constant $c>0$.

Taking all together there is a $0 \leq \varrho \leq r$ so that the following holds: $Z(s):=S(s) s^{-1}\left(s-\sigma_{\mathrm{a}}\right)^{\varrho}$ is holomorphic on $\Re s>\sigma_{\mathrm{a}}, Z^{(m)}(s)$ can be continued continuously to $\Re s \geq \sigma_{\mathrm{a}}$ and $\left|Z^{(m)}(s)\right| \ll_{m} \exp \left(c^{\prime}|\Im s|\right)$ for $\sigma_{\mathrm{a}} \leq$ $\Re s \leq \sigma_{\mathrm{a}}+1, m \in \mathbb{N}_{0}$ with a constant $c^{\prime}>0$. Let $\varrho \in \mathbb{N}_{0}$ be minimal with this property. According to Lemma 2.7, $S(s)$ is divergent at $s=\sigma_{\mathrm{a}}$. Hence $\lim _{\sigma \rightarrow \sigma_{\mathrm{a}}+0} S(\sigma)=\infty$. Therefore $\varrho \geq 1$. Furthermore, $c:=Z\left(\sigma_{\mathrm{a}}\right)=$ $\lim _{\sigma \rightarrow \sigma_{\mathrm{a}}+0} S(\sigma) \sigma^{-1}\left(\sigma-\sigma_{\mathrm{a}}\right)^{\varrho} \geq 0$. If $\Re s>\sigma_{\mathrm{a}}$ then $h_{s}(t):=Z\left(t s+(1-t) \sigma_{\mathrm{a}}\right)$ is a $C^{\infty}$-function on $(0,1]$. From the existence of the limit

$$
\lim _{t \rightarrow 0} h_{s}^{(m)}(t)=\left(s-\sigma_{\mathrm{a}}\right)^{m} \lim _{t \rightarrow 0} Z^{(m)}\left(t s+(1-t) \sigma_{\mathrm{a}}\right)=\left(s-\sigma_{\mathrm{a}}\right)^{m} Z^{(m)}\left(\sigma_{\mathrm{a}}\right)
$$

for $m \in \mathbb{N}_{0}$ it follows that $h \in C^{\infty}[0,1]$. Assume $c=0$. Then for $\Re s>\sigma_{\mathrm{a}}$ it would follow that

$$
\widetilde{Z}(s):=\frac{Z(s)}{s-\sigma_{\mathrm{a}}}=\int_{0}^{1} \frac{h_{s}^{\prime}(t)}{s-\sigma_{\mathrm{a}}} d t=\int_{0}^{1} Z^{\prime}\left(t s+(1-t) \sigma_{\mathrm{a}}\right) d t
$$


and for each $m \in \mathbb{N}_{0}$,

$$
\widetilde{Z}^{(m)}(s)=\int_{0}^{1} t^{m} Z^{(m+1)}\left(t s+(1-t) \sigma_{\mathrm{a}}\right) d t .
$$

Therefore $\widetilde{Z}(s)$ would be holomorphic on $\Re s>\sigma_{\mathrm{a}}, \widetilde{Z}^{(m)}(s)$ would be continuous on $\Re s \geq \sigma_{\mathrm{a}}$ and $\left|\widetilde{Z}^{(m)}(s)\right| \ll_{m} \exp \left(c^{\prime}|\Im s|\right)$ for $m \in \mathbb{N}_{0}, \sigma_{\mathrm{a}} \leq \Re s \leq$ $\sigma_{\mathrm{a}}+1$. This would contradict the minimality of $\varrho$. Therefore the assumption is false and $c>0$.

Let $\omega:=\varrho-1$ and for $\Re s>0$ set $H(s):=S\left(s+\sigma_{\mathrm{a}}\right)\left(s+\sigma_{\mathrm{a}}\right)^{-1}-c s^{-\omega-1}=$ $\left(Z\left(s+\sigma_{\mathrm{a}}\right)-Z\left(\sigma_{\mathrm{a}}\right)\right) s^{-\varrho}$. For $\Re s>0$ we have

$$
H^{\prime}(s)=\left(Z^{\prime}\left(s+\sigma_{\mathrm{a}}\right) s^{\varrho}-\left(Z\left(s+\sigma_{\mathrm{a}}\right)-Z\left(\sigma_{\mathrm{a}}\right)\right) \varrho s^{\varrho-1}\right) s^{-2 \varrho} .
$$

If $\varrho=1$ then by partial integration,

$$
\begin{aligned}
s^{2} H^{\prime}(s) & =h_{s+\sigma_{\mathrm{a}}}^{\prime}(1)-\left(h_{s+\sigma_{\mathrm{a}}}(1)-h_{s+\sigma_{\mathrm{a}}}(0)\right) \\
& =\int_{0}^{1} t h_{s+\sigma_{\mathrm{a}}}^{\prime \prime}(t) d t=s^{2} \int_{0}^{1} t Z^{\prime \prime}\left(t s+\sigma_{\mathrm{a}}\right) d t
\end{aligned}
$$

and therefore

$$
H^{\prime}(s)=\int_{0}^{1} t Z^{\prime \prime}\left(t s+\sigma_{\mathrm{a}}\right) d t \ll \exp \left(c^{\prime}|\Im s|\right) \quad \text { for } 0<\Re s \leq 1 .
$$

If $\varrho \geq 2$ then

$$
s^{2 \varrho} H^{\prime}(s)=Z^{\prime}\left(s+\sigma_{\mathrm{a}}\right) s^{\varrho}-\varrho s^{\varrho-1} \int_{0}^{1} h_{s+\sigma_{\mathrm{a}}}^{\prime}(t) d t
$$

and therefore

$$
\left|H^{\prime}(s)\right| \ll \frac{1}{|s|^{2 \varrho}}\left(|s|^{\varrho} e^{c^{\prime}|\Im s|}+|s|^{\varrho-1}|s| e^{c^{\prime}|\Im s|}\right) \ll \frac{1}{|s|^{\varrho}} e^{c^{\prime}|\Im s|}
$$

for $0<\Re s \leq 1$. We have $H(2 \sigma+i \tau)-H(\sigma+i \tau)=\int_{\sigma}^{2 \sigma} H^{\prime}(\alpha+i \tau) d \alpha$ for $1 / 2 \geq \sigma>0, \tau \in \mathbb{R}$. Consequently, for $\varrho=1$,

$$
|H(2 \sigma+i \tau)-H(\sigma+i \tau)| \ll \sigma e^{c^{\prime}|\tau|}
$$

and for $\varrho \geq 2$,

$$
|H(2 \sigma+i \tau)-H(\sigma+i \tau)| \ll \sigma \frac{1}{|\sigma+i \tau| \varrho} e^{c^{\prime}|\tau|} .
$$

For $0<\sigma \leq 1 / 2, T>0$, define

$$
\eta(\sigma, T):=\sigma^{\omega} \int_{-T}^{T}|H(2 \sigma+i \tau)-H(\sigma+i \tau)| d \tau .
$$


Then in the case $\varrho=1, \eta(\sigma, T) \ll \sigma \exp \left(c^{\prime} T\right)$ and in the case $\varrho \geq 2$ it follows by substitution $\tau=\sigma u$ that

$\eta(\sigma, T) \ll \sigma^{\omega+1} e^{c^{\prime} T} \int_{-T}^{T} \frac{1}{|\sigma+i \tau| \varrho} d \tau \ll \sigma^{\omega+1} e^{c^{\prime} T} \sigma^{1-\varrho} \int_{-\infty}^{\infty} \frac{d u}{|1+i u|^{\varrho}} \ll \sigma e^{c^{\prime} T}$.

Consequently, in both cases, $\lim _{\sigma \rightarrow 0} \eta(\sigma, T)=0$ for each $T>0$. Assume without loss of generality that $P(1, \ldots, 1)>1$. The effective Tauberian Theorem of Ikehara ([11], Theorem 11, p. 265) yields

$$
\sum_{P\left(n_{1}, \ldots, n_{r}\right) \leq e^{x}} \Lambda\left(n_{1}\right) \ldots \Lambda\left(n_{r}\right)=\left(\frac{c}{\Gamma(\omega+1)}+O(\varrho(x))\right) e^{\sigma_{\mathrm{a}} x} x^{\omega}
$$

as $x \rightarrow \infty$. Here $\varrho(x)=\inf _{T>0}\left(T^{-1}+\eta\left(x^{-1}, T\right)+(T x)^{-\omega-1}\right)$. Choosing $T=T(x)$ with $T^{-1}=x^{-1} e^{c^{\prime} T}$ gives $T(x) \asymp \log x$ and $\varrho(x) \ll \log ^{-1} x$. From this Theorem 1.5 follows.

6. Proof of Theorem 1.6. Choose $\Theta^{\prime}>0$ with $\varrho(P)+d(P) \Theta^{\prime}<\pi / 2$ and $a=1 / 2$. From Lemma 2.8 it follows that $|G(-z)| \ll|z|^{-1} \log (|z|+2)$ for $z \in \operatorname{Trace}\left(\gamma_{1}\right) \cup \operatorname{Trace}\left(\gamma_{-1}\right)$ or $|z|=(1+c) c^{n_{0}} / 2, n_{0} \in \mathbb{N}$. Consequently, for $\Re s>0$,

$$
\begin{aligned}
S(s) & :=\sum_{n_{1}, \ldots, n_{r} \geq 0} \frac{1}{P\left(c^{n_{1}}, \ldots, c^{n_{r}}\right)^{s}} \\
& =\frac{(-1)^{r}}{(2 \pi i)^{r}} \int_{\left(-\gamma_{1}+\gamma_{0}+\gamma_{-1}\right)^{r}} P(\underline{z})^{-s} \prod_{\varrho=1}^{r} G\left(-z_{\varrho}\right) d \underline{z} .
\end{aligned}
$$

The integral is absolutely convergent. Assume $b \in \mathbb{R}^{-} \backslash \mathbb{Z}$. For $z \in \operatorname{Trace}\left(\gamma_{1}\right) \cup$ $\operatorname{Trace}\left(\gamma_{0}\right) \cup \operatorname{Trace}\left(\gamma_{-1}\right)$ set

$$
H(z):=G(-z)+(z \log c)^{-1} \log (-z) .
$$

Then $H(z)$ is continuous everywhere with the exception of the point $a$. From Lemma 2.8 it follows that for $z \in \operatorname{Trace}\left(\gamma_{1}\right) \cup \operatorname{Trace}\left(\gamma_{-1}\right)$,

$$
\begin{aligned}
H(z)= & -\frac{1}{2 z}-\sum_{[b]+1 \leq m \leq-1}\left(1-c^{-m}\right)^{-1} z^{m-1} \\
& -\frac{\pi}{z \log c} \sum_{0 \neq n \in \mathbb{Z}} \sin \left(\frac{2 \pi^{2} i n}{\log c}\right)^{-1} \exp \left(\frac{2 \pi i n \log (-z)}{\log c}\right)+R(z)
\end{aligned}
$$

where $|R(z)| \ll_{b, \Theta^{\prime}}|z|^{b-1}$. The $n$th summand is $\ll \exp \left(-2 \pi|n| \Theta^{\prime} / \log c\right)$ and therefore the $n$-series has a convergent majorant on $\operatorname{Trace}\left(\gamma_{1}\right) \cup \operatorname{Trace}\left(\gamma_{-1}\right)$ which is independent of $z$. Expressing $G$ in (6.1) by $H$ and using an argument 
similar to (3.4) one sees that $S(s)$ is a linear combination of the integrals

$$
\begin{aligned}
\int_{\left(-\gamma_{1}+\gamma_{0}+\gamma_{-1}\right)^{\left|\bar{K}_{0}\right|}} d\left(z_{\varrho}\right)_{\varrho \in \bar{K}_{0}} & \prod_{\varrho \in \bar{K}_{0}} H\left(z_{\varrho}\right) \\
& \times \int_{[a, \infty)^{\left|K_{0}\right|}} d\left(t_{\varrho}\right)_{\varrho \in K_{0}} \prod_{\varrho \in K_{0}} t_{\varrho}^{-1} P\left(\left(t_{\varrho}\right)_{\varrho \in K_{0}},\left(z_{\varrho}\right)_{\varrho \in \bar{K}_{0}}\right)^{-s}
\end{aligned}
$$

where $K_{0}$ runs through all subsets of $\{1, \ldots, r\}$ and $\bar{K}_{0}:=\{1, \ldots, r\} \backslash K_{0}$. Decomposing the lines of integration $-\gamma_{1}+\gamma_{0}+\gamma_{-1}$ and parameterizing $\gamma_{ \pm 1}$ shows with (6.2) that $S(s)$ is a linear combination of the integrals

$$
\begin{aligned}
& \quad \int_{\gamma_{0}^{\left|K_{1}\right|}} d\left(z_{\varrho}\right)_{\varrho \in K_{1}} \prod_{\varrho \in K_{1}} H\left(z_{\varrho}\right) \int_{[1, \infty)^{\left|K_{0}\right|}} d\left(t_{\varrho}\right)_{\varrho \in K_{0}} \prod_{\varrho \in K_{0}} t_{\varrho}^{-1} \\
& \times \int_{[1, \infty)^{\left|K_{2}\right|}} d\left(t_{\varrho}\right)_{\varrho \in K_{2}} \prod_{\varrho \in K_{2}} t_{\varrho}^{m_{\varrho}-1} \int_{[1, \infty)^{\left|K_{3}\right|}} d\left(t_{\varrho}\right)_{\varrho \in K_{3}} \\
& \times \prod_{\varrho \in K_{3}} t_{\varrho}^{-1}\left(\sum_{0 \neq n_{\varrho} \in \mathbb{Z}} \sin \left(\frac{2 \pi^{2} i n_{\varrho}}{\log c}\right)^{-1} \exp \left(\frac{2 \pi i n_{\varrho}}{\log c} \log \left(-t_{\varrho} \frac{a}{\cos \Theta^{\prime}} e^{i j_{\varrho} \Theta^{\prime}}\right)\right)\right) \\
& \times \int_{[1, \infty)^{\left|K_{4}\right|}} d\left(t_{\varrho}\right)_{\varrho \in K_{4}} \prod_{\varrho \in K_{4}} R\left(t_{\varrho} \frac{a}{\cos \Theta^{\prime}} e^{i j_{\varrho} \Theta^{\prime}}\right) \\
& \times P\left(\left(z_{\varrho}\right)_{\varrho \in K_{1}},\left(t_{\varrho} \frac{a}{\cos \Theta^{\prime}} e^{i j_{\varrho} \Theta^{\prime}}\right)_{\varrho \in \bar{K}_{1}},\left(a t_{\varrho}\right)_{\varrho \in K_{0}}\right)^{-s}
\end{aligned}
$$

where $\left(K_{1}, K_{2}, K_{3}, K_{4}\right)$ runs through all disjoint decompositions $\bar{K}_{0}=K_{1} \dot{\cup}$ $K_{2} \cup K_{3} \cup K_{4}, \bar{K}_{1}:=K_{2} \cup K_{3} \cup K_{4},\left(j_{\varrho}\right)_{\varrho \in \bar{K}_{1}}$ runs through $\{ \pm 1\}^{\left|\bar{K}_{1}\right|}$ and $\left(m_{\varrho}\right)_{\varrho \in K_{2}}$ runs through $\{[b]+1, \ldots, 0\}^{\left|K_{2}\right|}$. For $\Re s>0$ it follows from (3.5) that $\left|P(\underline{z})^{-s}\right| \ll_{s}\left|z_{1} \ldots z_{r}\right|^{-\Re s / \sigma_{\mathrm{a}}^{*}}$ for $\underline{z} \in S_{\Theta^{\prime}, a}^{r}$. Each of the $n_{\varrho^{-}}$-series in (6.3) has a convergent majorant which is independent of $t_{\varrho}$ and the other factors of the integrand are together $\ll \prod_{\varrho \in K_{0} \cup \bar{K}_{1}} t_{\varrho}^{-1-\Re s / \sigma_{a}^{*}}$. Therefore integration and the $n_{\varrho}$-summations in (6.3) may be interchanged. Theorem 2.1 shows that each of the integrals (6.3) is a linear combination of the functions

$$
\begin{aligned}
I(s)= & \sum_{\substack{0 \neq n_{\varrho} \in \mathbb{Z} \\
\varrho \in K_{3}}} \prod_{\varrho \in K_{3}} \sin \left(\frac{2 \pi^{2} i n_{\varrho}}{\log c}\right)^{-1} \exp \left(\frac{2 \pi n_{\varrho} j_{\varrho}}{\log c}\left(\pi-\Theta^{\prime}\right)\right) \\
& \times\left(\frac{a}{\cos \Theta^{\prime}}\right)^{2 \pi i n_{\varrho} / \log c} \int_{\gamma_{0}^{\left|K_{1}\right|}} d\left(z_{\varrho}\right)_{\varrho \in K_{1}} \prod_{\varrho \in K_{1}} H\left(z_{\varrho}\right)
\end{aligned}
$$




$$
\begin{aligned}
& \times \int_{[1, \infty)^{\left|K_{0}\right|+\left|\bar{K}_{1}\right|}} d\left(\tau_{\mu}\right)_{\mu \in K_{0} \cup \bar{K}_{1}} \prod_{\varrho \in K_{0}}\left(\prod_{\mu \in K_{0} \cup \bar{K}_{1}} \tau_{\mu}^{\lambda_{\mu \varrho}}\right)^{-1} \\
& \times \prod_{\varrho \in K_{2}}\left(\prod_{\mu \in K_{0} \cup \bar{K}_{1}} \tau_{\mu}^{\lambda_{\mu \varrho}}\right)^{m_{\varrho}-1} \prod_{\varrho \in K_{3}}\left(\prod_{\mu \in K_{0} \cup \bar{K}_{1}} \tau_{\mu}^{\lambda_{\mu \varrho}}\right)^{-1+\frac{2 \pi i n_{\varrho}}{\log c}} \\
& \times \prod_{\varrho \in K_{4}} R\left(\prod_{\mu \in K_{0} \cup \bar{K}_{1}} \tau_{\mu}^{\lambda_{\mu}} \frac{a}{\cos \Theta^{\prime}} e^{i j_{\varrho} \Theta^{\prime}}\right) \\
& \times \prod_{\varrho \in K_{0} \cup \bar{K}_{1}} \tau_{\varrho}^{-1} \prod_{\mu \in K_{0} \cup \bar{K}_{1}} \tau_{\mu}^{\lambda_{\mu \varrho}} Q\left(\left(z_{\varrho}\right)_{\varrho \in K_{1}},\left(\tau_{\mu}\right)_{\mu \in K_{0} \cup \bar{K}_{1}}\right)^{-s}
\end{aligned}
$$

where $\underline{\underline{\lambda}}=\left(\lambda_{\mu \varrho}\right) \in \mathbb{N}_{0}^{\left(\left|K_{0}\right|+\left|\bar{K}_{1}\right|\right)^{2}}$ runs through finitely many regular matrices and $Q$ runs through finitely many polynomials of the form

$$
Q\left(\left(Z_{\varrho}\right)_{\varrho \in K_{1}},\left(T_{\mu}\right)_{\mu \in K_{0} \cup \bar{K}_{1}}\right)=\sum_{\underline{\gamma} \leq \underline{\gamma}^{\prime}} b_{\underline{\gamma}}(\underline{Z}) \prod_{\mu \in K_{0} \cup \bar{K}_{1}} T_{\mu}^{\gamma_{\mu}} .
$$

Here $b_{\underline{\gamma}}(\underline{Z})$ is a polynomial in $\underline{Z}$ and $\Re b_{\underline{\gamma}}(\underline{z})>0$ for $b_{\underline{\gamma}} \not \equiv 0$ and $\underline{z} \in \gamma_{0}^{\left|K_{1}\right|}$; furthermore, $b_{\gamma^{\prime}} \not \equiv 0$ and $\gamma_{\mu}^{\prime} \geq 1$ for $\mu \in K_{0} \cup \bar{K}_{1}$. Substitution of $\tau_{\mu}^{-1}$ for $\tau_{\mu}$ with $\mu \in \widehat{K_{0}} \cup \bar{K}_{1}$ gives

(6.5) $\quad I(s)$

$$
\begin{aligned}
= & \sum_{\substack{0 \neq n_{\varrho} \in \mathbb{Z} \\
\varrho \in K_{3}}} \prod_{\varrho \in K_{3}} \sin \left(\frac{2 \pi^{2} i n_{\varrho}}{\log c}\right)^{-1} \exp \left(\frac{2 \pi n_{\varrho} j_{\varrho}}{\log c}\left(\pi-\Theta^{\prime}\right)\right)\left(\frac{a}{\cos \Theta^{\prime}}\right)^{2 \pi i n_{\varrho} / \log c} \\
& \times \int_{\gamma_{0}^{\left|K_{1}\right|}} d\left(z_{\varrho}\right)_{\varrho \in K_{1}} \prod_{\varrho \in K_{1}} H\left(z_{\varrho}\right) \int_{(0,1)^{|U|}} d\left(\tau_{\mu}\right)_{\mu \in U} \prod_{\mu \in U} \tau_{\mu}^{\alpha_{\mu}+\gamma_{\mu}^{\prime} s} \widetilde{R}\left(\left(\tau_{\mu}\right)_{\mu \in U}\right) \\
& \times \int_{(0,1)^{|V|}} d\left(\tau_{\mu}\right)_{\mu \in V} \prod_{\mu \in V} \tau_{\mu}^{\alpha_{\mu}+\gamma_{\mu}^{\prime} s}\left(\sum_{\underline{\gamma} \leq \underline{\gamma}^{\prime}} b_{\underline{\gamma}}\left(\left(z_{\varrho}\right)_{\varrho \in K_{1}}\right) \prod_{\mu \in K_{0} \cup \bar{K}_{1}} \tau_{\mu}^{\gamma_{\mu}^{\prime}-\gamma_{\mu}}\right)^{-s}
\end{aligned}
$$

where $U:=\left\{\mu \in K_{0} \cup \bar{K}_{1} \mid \lambda_{\mu \varrho} \geq 1\right.$ for a $\left.\varrho \in K_{4}\right\}, V:=\left(K_{0} \cup \bar{K}_{1}\right) \backslash U$, and for each $\mu \in K_{0} \cup \bar{K}_{1}$,

$$
\begin{aligned}
\alpha_{\mu}= & \alpha_{\mu}\left(\left(n_{\varrho}\right)_{\varrho \in K_{3}}\right) \\
:= & \sum_{\varrho \in K_{0}} \lambda_{\mu \varrho}+\sum_{\varrho \in K_{2}} \lambda_{\mu \varrho}\left(1-m_{\varrho}\right) \\
& +\sum_{\varrho \in K_{3}} \lambda_{\mu \varrho}\left(1-\frac{2 \pi i n_{\varrho}}{\log c}\right)-\sum_{\varrho \in K_{0} \cup \bar{K}_{1}} \lambda_{\mu \varrho}-1 .
\end{aligned}
$$


For $\left(\tau_{\mu}\right)_{\mu \in U} \in(0,1)^{|U|}$ we have

$$
\begin{aligned}
\widetilde{R}\left(\left(\tau_{\mu}\right)_{\mu \in U}\right) & :=\prod_{\varrho \in K_{4}} R\left(\prod_{\mu \in U} \tau_{\mu}^{-\lambda_{\mu} \varrho} \frac{a}{\cos \Theta^{\prime}} e^{i j_{\varrho} \Theta^{\prime}}\right) \\
& \ll b, \Theta^{\prime} \prod_{\varrho \in K_{4}}\left(\prod_{\mu \in U} \tau_{\mu}^{-\lambda_{\mu \varrho}}\right)^{b-1} \\
& \ll \prod_{\mu \in U} \tau_{\mu}^{(1-b) \sum_{\varrho \in K_{4}} \lambda_{\mu \varrho}} .
\end{aligned}
$$

For $\mu \in V$,

$$
\Re \alpha_{\mu} \geq \sum_{\varrho \in K_{0} \cup K_{2} \cup K_{3}} \lambda_{\mu \varrho}-\sum_{\varrho \in K_{0} \cup \bar{K}_{1}} \lambda_{\mu \varrho}-1=-1 .
$$

Furthermore, for $\left(\tau_{\mu}\right)_{\mu \in U} \in(0,1)^{|U|}$,

$$
\begin{aligned}
\prod_{\mu \in U} \tau_{\mu}^{\alpha_{\mu}} \widetilde{R}\left(\left(\tau_{\mu}\right)_{\mu \in U}\right) & \ll \prod_{\mu \in U} \tau_{\mu}^{\sum_{\varrho \in K_{0} \cup K_{2} \cup K_{3}} \lambda_{\mu \varrho}-\sum_{\varrho \in K_{0} \cup \bar{K}_{1}} \lambda_{\mu \varrho}-1+(1-b) \sum_{\varrho \in K_{4}} \lambda_{\mu \varrho}} \\
& \ll \prod_{\mu \in U} \tau_{\mu}^{-1-b} .
\end{aligned}
$$

Therefore the triple integrals in (6.5) are absolutely convergent for $\Re s>0$. From Lemma 2.5 it follows with $\varepsilon>0$ which depends only on $Q$ that the innermost integral in (6.5) is for $\Re s>0, \gamma_{\mu}^{\prime} s+\alpha_{\mu} \notin \mathbb{Z}, \mu \in V$ equal to

$$
\begin{aligned}
& \prod_{\mu \in V}\left(e\left(\gamma_{\mu}^{\prime} s+\alpha_{\mu}\right)-1\right)^{-1} \\
& \quad \times \int_{I_{\varepsilon}^{|V|}} \prod_{\mu \in V} v_{\mu}^{\alpha_{\mu}+\gamma_{\mu}^{\prime} s}\left(\sum_{\underline{\gamma} \leq \underline{\gamma}^{\prime}} b_{\underline{\gamma}}\left(\left(z_{\varrho}\right)_{\varrho \in K_{1}}\right) \prod_{\mu \in U} \tau_{\mu}^{\gamma_{\mu}^{\prime}-\gamma_{\mu}} \prod_{\mu \in V} v_{\mu}^{\gamma_{\mu}^{\prime}-\gamma_{\mu}}\right)^{-s} d\left(v_{\mu}\right)_{\mu \in V} .
\end{aligned}
$$

Substituting this sum into (6.5) gives for $\Re s>0, \gamma_{\mu}^{\prime} s \notin \mathbb{Z}+(2 \pi i / \log c) \mathbb{Z}$, $\mu \in V$, the representation

where

$$
I(s)=\sum_{\substack{0 \neq n_{\varrho} \in \mathbb{Z} \\ \varrho \in K_{3}}} A\left(s ;\left(n_{\varrho}\right)_{\varrho \in K_{3}}\right) B\left(s ;\left(n_{\varrho}\right)_{\varrho \in K_{3}}\right)
$$

$$
\begin{aligned}
& B(s, \underline{n}) \\
& :=\int_{\gamma_{0}^{\left|K_{1}\right|}} d\left(z_{\varrho}\right)_{\varrho \in K_{1}} \prod_{\varrho \in K_{1}} H\left(z_{\varrho}\right) \int_{(0,1)^{|U|}} d\left(\tau_{\mu}\right)_{\mu \in U} \prod_{\mu \in U} \tau_{\mu}^{\alpha_{\mu}+\gamma_{\mu}^{\prime} s} \widetilde{R}\left(\left(\tau_{\mu}\right)_{\mu \in U}\right) \\
& \quad \times \int_{I_{\varepsilon}^{|V|}} d\left(v_{\mu}\right)_{\mu \in V} \prod_{\mu \in V} v_{\mu}^{\alpha_{\mu}+\gamma_{\mu}^{\prime} s}\left(\sum_{\underline{\gamma} \leq \underline{\gamma}^{\prime}} b_{\gamma}\left(\left(z_{\varrho}\right)_{\varrho \in K_{1}}\right) \prod_{\mu \in U} \tau_{\mu}^{\gamma_{\mu}^{\prime}-\gamma_{\mu}} \prod_{\mu \in V} v_{\mu}^{\gamma_{\mu}^{\prime}-\gamma_{\mu}}\right)^{-s}
\end{aligned}
$$


can be continued holomorphically to $\Re s>\sigma(b):=b \min _{\mu \in U} 1 / \gamma_{\mu}^{\prime}$. For each $\sigma(b)<c_{1} \leq \Re s \leq c_{2},|\Im s| \leq c_{3},|B(s ; \underline{n})| \ll_{c_{1}, c_{2}, c_{3}} 1$ uniformly in $\underline{n}$.

Furthermore,

$$
\begin{aligned}
A(s, \underline{n}):= & \prod_{\varrho \in K_{3}} \sin \left(\frac{2 \pi^{2} i n_{\varrho}}{\log c}\right)^{-1} \exp \left(\frac{2 \pi n_{\varrho} j_{\varrho}}{\log c}\left(\pi-\Theta^{\prime}\right)\right)\left(\frac{a}{\cos \Theta^{\prime}}\right)^{2 \pi i n_{\varrho} / \log c} \\
& \times \prod_{\mu \in V}\left(e\left(\gamma_{\mu}^{\prime} s+\alpha_{\mu}\right)-1\right)^{-1}
\end{aligned}
$$

is meromorphic on $\mathbb{C}$. As $\alpha_{\mu}+\sum_{\varrho \in K_{3}} \lambda_{\mu \varrho} 2 \pi i n_{\varrho} / \log c \in \mathbb{Z}$ each of the poles of $A(s)$ lies in $N^{-1} \mathbb{Z}+2 \pi i(N \log c)^{-1} \mathbb{Z}$ where $N:=\operatorname{lcm}\left\{\gamma_{\mu}^{\prime} \mid \mu \in\right.$ $V\} \in \mathbb{N}$. This set is independent of $\left(n_{\varrho}\right)_{\varrho \in K_{3}}$. Therefore on summation over $\left(n_{\varrho}\right)_{\varrho \in K_{3}}$ no accumulation of poles in $\mathbb{C}$ can occur. For the more general series $\sum_{n_{1}, \ldots, n_{r} \geq 0} P\left(c_{1}^{n_{1}}, \ldots, c_{r}^{n_{r}}\right)^{-s}$ such an accumulation cannot be ruled out. Therefore it cannot be continued meromorphically to $\mathbb{C}$ with the method of this paper.

For $R \geq 1$ define

$$
F_{R}(s):=\prod_{\substack{(k, l) \in \mathbb{Z}^{2}: \\|k / N+2 \pi i l /(N \log c)| \leq 2 R}}\left(s-\frac{k}{N}-\frac{2 \pi i l}{N \log c}\right)^{|V|} \in \mathbb{C}[s] .
$$

Then $F_{R}(s) A(s, \underline{n})$ has no poles in the disk $K_{R}(0)$ with center 0 and radius $R$. For $s \in K_{R}(0)$,

$$
\begin{aligned}
& \left|F_{R}(s) A(s, \underline{n})\right| \ll \prod_{\varrho \in K_{3}} \exp \left(-\frac{2 \pi\left|n_{\varrho}\right|}{\log c} \Theta^{\prime}\right) \\
\times & \prod_{\mu \in V}\left\{\left|e\left(\gamma_{\mu}^{\prime} s+\alpha_{\mu}\right)-1\right|^{-1} \prod_{\substack{(k, l) \in \mathbb{Z}^{2}: \\
|k / N+2 \pi i l /(N \log c)| \leq 2 R}}\left|s-\frac{k}{N}-\frac{2 \pi i l}{N \log c}\right|\right\} .
\end{aligned}
$$

Assume $\mu \in V$ and let the minimum

$$
\delta:=\min _{k \in \mathbb{Z}}\left|\gamma_{\mu}^{\prime} s-\sum_{\varrho \in K_{3}} \lambda_{\mu \varrho} 2 \pi i n_{\varrho}(\log c)^{-1}-k\right|
$$

be attained at $k=k_{0}$. If $\delta \leq 1 / 2$ then define $k_{1}:=k_{0} N \gamma_{\mu}^{\prime-1}, l_{1}:=$ $\sum_{\varrho \in K_{3}} \lambda_{\mu \varrho} n_{\varrho} N \gamma_{\mu}^{\prime-1} \in \mathbb{Z}$. Then $\left|\gamma_{\mu}^{\prime} s-k_{0}-2 \pi i l_{1} \gamma_{\mu}^{\prime} /(N \log c)\right|=\delta \leq 1 / 2$ and $\left|k_{1} / N+2 \pi i l_{1} /(N \log c)\right| \leq|s|+1 /\left(2 \gamma_{\mu}^{\prime}\right) \leq 2 R$. The $\mu$ th factor in (6.6) is therefore

$$
\left|s-\frac{k}{N}-\frac{2 \pi i l}{N \log c}\right| \frac{\left|\gamma_{\mu}^{\prime} s-k_{0}-2 \pi i l_{1} \gamma_{\mu}^{\prime} /(N \log c)\right|}{\gamma_{\mu}^{\prime}\left|e\left(\gamma_{\mu}^{\prime} s-k_{0}-2 \pi i l_{1} \gamma_{\mu}^{\prime} /(N \log c)\right)-1\right|}
$$$$
\ll(R+2 R)^{2 R N R N(\log c) / \pi} \gamma_{\mu}^{\prime-1} \ll_{R} 1 .
$$ 
If $\delta>1 / 2$ then the $\mu$ th factor in (6.6) is

$$
\ll(3 R)^{2 R N R N(\log c) / \pi}\left|e\left(\gamma_{\mu}^{\prime} s+\alpha_{\mu}\right)-1\right|^{-1} \ll_{R} 1 .
$$

Therefore $\left|F_{R}(s) A(s, \underline{n})\right| \ll_{R} \prod_{\varrho \in K_{3}} \exp \left(-2 \pi \Theta^{\prime}\left|n_{\varrho}\right| / \log c\right)$ for $s \in K_{R}(0)$ where the $\ll$-constant is independent of $\left(n_{\varrho}\right)_{\varrho \in K_{3}}$. Consequently, in the representation

$$
F_{R}(s) I(s)=\sum_{\substack{0 \neq n_{\varrho} \in \mathbb{Z} \\ \varrho \in K_{3}}} F_{R}(s) A\left(s,\left(n_{\varrho}\right)_{\varrho \in K_{3}}\right) B\left(s,\left(n_{\varrho}\right)_{\varrho \in K_{3}}\right)
$$

which is valid on $\left\{s \in \mathbb{C}|\Re s>0| s \mid,<R, s \notin N^{-1} \mathbb{Z}+2 \pi i(N \log c)^{-1} \mathbb{Z}\right\}$ each summand is holomorphic on

$$
\{s \in \mathbb{C}|\Re s>\sigma(b),| s \mid<R\}
$$

and the series is uniformly convergent on each compact subset of (6.7). $F_{R}(s) I(s)$ can therefore be continued holomorphically to $(6.7)$ and so $I(s)$ can be continued meromorphically to $(6.7)$ where the poles lie in $N^{-1} \mathbb{Z}+$ $(2 \pi i /(N \log c)) \mathbb{Z}$ and are of order $\leq|V| \leq r$. As $R \geq 1$ may be chosen arbitrarily $I(s)$ can be continued meromorphically to $\Re s>\sigma(b)$ with the same restrictions on the poles as above. As $S(s)$ is a linear combination of integrals of the type $I(s)$ it follows that $S(s)$ can be continued meromorphically to $\Re s>c b$ with poles lying in $\left(1 / N^{*}\right) \mathbb{Z}+\left(2 \pi i /\left(N^{*} \log c\right)\right) \mathbb{Z}$ and order $\leq r$. Here $c>0$ and $N^{*} \in \mathbb{N}$ depend only on the finitely many integral matrices $\underline{\underline{\lambda}}$ and the associated maximal exponent vectors $\gamma^{\prime}$. Therefore $c$ and $N^{*}$ depend only on $P$. As $b \in \mathbb{R}^{-} \backslash \mathbb{Z}$ can be chosen arbitrarily $S(s)$ can be continued meromorphically to $\mathbb{C}$ with the same restrictions on the poles as above. For $\Re s>0,\left|P\left(c^{n_{1}}, \ldots, c^{n_{r}}\right)^{-s}\right| \ll e^{|\Im s| \varrho(P)} c^{-\Re s\left(n_{1}+\ldots+n_{r}\right) / \sigma_{\mathrm{a}}^{*}}$. Therefore the Dirichlet series $S(s)$ is uniformly convergent on each compact subset of $\Re s>0$ where it defines a holomorphic function. The poles of $S(s)$ lie consequently in $\left(-1 / N^{*}\right) \mathbb{N}_{0}+\left(2 \pi i /\left(N^{*} \log c\right)\right) \mathbb{Z}$.

7. Proof of Corollary 1.7. For $t \in \mathbb{R}$ define $A(t):=\#\left\{\left(n_{1}, \ldots, n_{r}\right) \in\right.$ $\left.\mathbb{N}_{0}^{r} \mid P\left(c^{n_{1}}, \ldots, c^{n_{r}}\right) \leq e^{t}\right\}$. Then $A(t) \leq \#\left\{\left(n_{1}, \ldots, n_{r}\right) \in \mathbb{N}_{0}^{r} \mid c^{\left(n_{1}+\ldots+n_{r}\right) / \sigma_{\mathrm{a}}^{*}}\right.$ $\left.\ll e^{t}\right\} \ll t^{r}+1$ for $t \geq 0$. Assume without loss of generality that $P(1, \ldots, 1)$ $>1$. By partial integration and summation it follows that for $\Re s>0$ (1.7) has the representation

$$
S(s)=\int_{0}^{\infty} e^{-s t} d A(t)
$$

From Theorem 1.6 it follows that $S(s)$ is meromorphic on $\mathbb{C}$. As the Dirichlet series representation of $S(s)$ is divergent at $s=0$ we have $\lim _{\sigma \rightarrow 0+0} S(\sigma)=$ $\infty$ and consequently $S(s)$ has a pole at $s=0$. Let $1 \leq \omega \leq r$ be its order. Then there is a $C \in \mathbb{C} \backslash\{0\}$ with $s^{\omega} S(s)=C+O(|s|)$ as $s \rightarrow 0$. Hence 
$C=\lim _{\sigma \rightarrow 0+0} \sigma^{\omega} S(\sigma) \geq 0$ and therefore even $C>0$. Application of the Tauberian theorem with explicit remainder term of Karamata-Freud ([11], Theorem 9, p. 257, $\psi(t):=t$ ) gives

$$
A(x)=\left(C+O\left((\log x)^{-1}\right)\right) x^{\omega} / \Gamma(\omega+1) \quad \text { as } x \rightarrow \infty .
$$

8. Proof of Theorem 1.8. The proof is completely analogous to that of Theorem 1.6 where (6.2) is substituted by

$$
\begin{aligned}
G_{1}(-z):= & \sum_{n \geq 1}\left(\frac{\Lambda(n)}{n-z}-\frac{\Lambda(n)}{n}\right) \\
= & \sum_{[b]+1 \leq n \leq 1}(-z)^{n-1}\left(a_{n} \log (-z)+b_{n}\right) \\
& -\sum_{\varrho} \frac{\pi}{\sin \pi \varrho}(-z)^{\varrho-1}+R_{1}(z)
\end{aligned}
$$

with $R_{1}(z) \ll_{b, \Theta^{\prime}}|z|^{b-1}$ for $z \in \operatorname{Trace}\left(\gamma_{1}\right) \cup \operatorname{Trace}\left(\gamma_{-1}\right)$. These asymptotics follow from Lemma 2.6. The $\varrho$ th summand is $\ll e^{-\pi|\Im \varrho|}|z|^{\Re \varrho-1} e^{|\Im \varrho|\left(\pi-\Theta^{\prime}\right)}$ $\ll e^{-\Theta^{\prime}|\Im \varrho|}$. Define $H(z)$ to be the sum of the last two terms in (8.1). Furthermore, the functions

$$
\begin{aligned}
G_{\varrho}(z) & :=\sum_{0 \neq n \in \mathbb{Z}}\left(\frac{1}{z-n}+\frac{1}{n}\right)+\frac{1}{z}=\pi \cot \pi z \\
& =i \pi+\frac{2 \pi i}{e(z)-1}=-i \pi+\frac{2 \pi i}{1-e(-z)}, \quad 2 \leq \varrho \leq r,
\end{aligned}
$$

are used for which the asymptotics

$$
\begin{aligned}
G_{\varrho}(-z) & =j i \pi+R_{\varrho}(z), \\
R_{\varrho}(z) & =O\left(e^{-2 \pi|\Im z|}\right), \quad z \in \operatorname{Trace}\left(\gamma_{j}\right), \quad j= \pm 1,
\end{aligned}
$$

hold. As in the proof of Theorem 1.6 one shows that for $\Re s>\sigma_{\mathrm{a}}^{*}$ (see (3.5)) the function

$$
\begin{aligned}
S(s) & :=\sum_{n_{1}, \ldots, n_{r} \geq 1} \frac{\Lambda\left(n_{1}\right)}{P\left(n_{1}, \ldots, n_{r}\right)^{s}} \\
& =\frac{(-1)^{r}}{(2 \pi i)^{r}} \int_{\left(-\gamma_{1}+\gamma_{0}+\gamma_{-1}\right)^{r}} P(\underline{z})^{-s} \prod_{\varrho=1}^{r} G_{\varrho}\left(-z_{\varrho}\right) d \underline{z}
\end{aligned}
$$

can be represented as a linear combination of the following types of functions: 
- Type I:

$$
\begin{aligned}
& \prod_{\mu \in V}\left(e\left(\gamma_{\mu}^{\prime} s\right)-1\right)^{-1} \int_{\gamma_{0}^{\left|K_{0}\right|+1}} d\left(z_{\varrho}\right)_{\varrho \in K_{0} \cup\{1\}} H\left(z_{1}\right) \prod_{\varrho \in K_{0}} G_{\varrho}\left(-z_{\varrho}\right) \\
& \times \int_{(0,1)^{|U|}} d\left(\tau_{\mu}\right)_{\mu \in U} \widetilde{R}\left(\left(\tau_{\mu}\right)_{\mu \in U}\right) \prod_{\mu \in U} \tau_{\mu}^{\gamma_{\mu}^{\prime} s-\sum_{\varrho \in K_{1} \cup K_{2}} \lambda_{\mu \varrho}-1} \\
& \times \int_{I_{\varepsilon}^{|V|}} \prod_{\mu \in V} v_{\mu}^{\gamma_{\mu}^{\prime} s-\sum_{\varrho \in K_{1} \cup K_{2}} \lambda_{\mu \varrho}-1} \\
& \times\left(\sum_{\underline{\gamma} \leq \underline{\gamma}^{\prime}} b_{\underline{\gamma}}\left(\left(z_{\varrho}\right)_{\varrho \in K_{0} \cup\{1\}}\right) \prod_{\mu \in U} \tau_{\mu}^{\gamma_{\mu}^{\prime}-\gamma_{\mu}} \prod_{\mu \in V} v_{\mu}^{\gamma_{\mu}^{\prime}-\gamma_{\mu}}\right)^{-s} d\left(v_{\mu}\right)_{\mu \in V},
\end{aligned}
$$

where $K_{0} \dot{\cup} K_{1} \dot{\cup} K_{2}=\{2, \ldots, r\}, \underline{\underline{\lambda}} \in \mathbb{N}_{0}^{\left(\left|K_{1}\right|+\left|K_{2}\right|\right)^{2}}$ regular, $U:=\{\mu \in$ $K_{1} \cup K_{2} \mid \lambda_{\mu \varrho} \geq 1$ for a $\left.\varrho \in K_{2}\right\}, V:=\left(K_{1} \cup K_{2}\right) \backslash U, \varepsilon>0$ appropriately chosen and $\widetilde{R}:(0,1)^{|U|} \rightarrow \mathbb{C}$ is continuous and

$$
\widetilde{R}\left(\left(\tau_{\mu}\right)_{\mu \in U}\right) \ll \exp \left(-c \sum_{\mu \in U} \tau_{\mu}^{-1}\right)
$$

with a constant $c>0$;

- Type II:

$$
\begin{aligned}
& \prod_{\mu \in V}\left(e\left(\gamma_{\mu}^{\prime} s\right)-1\right)^{-1} \int_{\gamma_{0}^{\left|K_{0}\right|}} d\left(z_{\varrho}\right)_{\varrho \in K_{0}} \prod_{\varrho \in K_{0}} G_{\varrho}\left(-z_{\varrho}\right) \\
& \times \int_{(0,1)^{|U|}} d\left(\tau_{\mu}\right)_{\mu \in U} \prod_{\mu \in U} \tau_{\mu}^{\gamma_{\mu}^{\prime} s-\lambda_{\mu 1}(n-1)-1-\sum_{\varrho \in K_{1} \cup K_{2} \cup\{1\}} \lambda_{\mu \varrho}} \widetilde{R}\left(\left(\tau_{\mu}\right)_{\mu \in U}\right) \\
& \times \int_{I_{\varepsilon}^{|V|}} d\left(v_{\mu}\right)_{\mu \in V} \prod_{\mu \in V} v_{\mu}^{\gamma_{\mu}^{\prime} s-\lambda_{\mu 1}(n-1)-1-\sum_{\varrho \in K_{1} \cup K_{2} \cup\{1\}} \lambda_{\mu \varrho}} \\
& \times\left(\sum_{\underline{\gamma} \leq \underline{\gamma}^{\prime}} b_{\underline{\gamma}}\left(\left(z_{\varrho}\right)_{\varrho \in K_{0}}\right) \prod_{\mu \in U} \tau_{\mu}^{\gamma_{\mu}^{\prime}-\gamma_{\mu}} \prod_{\mu \in V} v_{\mu}^{\gamma_{\mu}^{\prime}-\gamma_{\mu}}\right)^{-s},
\end{aligned}
$$

where $K_{0} \dot{\cup} K_{1} \dot{\cup} K_{2}=\{2, \ldots, r\}, \lambda \in \mathbb{N}_{0}^{\left(\left|K_{1}\right|+\left|K_{2}\right|+1\right)^{2}}$ regular, $[b]+1 \leq n \leq 1$, $U:=\left\{\underset{\widetilde{R}}{\mu} \in K_{1} \cup K_{2} \cup\{1\} \mid \lambda_{\mu \varrho} \geq \overline{\overline{1}}\right.$ for a $\left.\varrho \in K_{2}\right\}, V:=\left(K_{1} \cup K_{2} \cup\{1\}\right) \backslash U$, $\varepsilon$ and $\widetilde{R}$ as above; 
- Type III:

$$
\begin{aligned}
\prod_{\mu \in V}\left(e\left(\gamma_{\mu}^{\prime} s\right)-1\right)^{-1} \int_{\gamma_{0}^{\left|K_{0}\right|}} d\left(z_{\varrho}\right)_{\varrho \in K_{0}} \prod_{\varrho \in K_{0}} G_{\varrho}\left(-z_{\varrho}\right) \\
\quad \times \int_{(0,1)^{|U|}} d\left(\tau_{\mu}\right)_{\mu \in U} \widetilde{R}\left(\left(\tau_{\mu}\right)_{\mu \in U}\right) \prod_{\mu \in U} \tau_{\mu}^{\gamma_{\mu}^{\prime} s-\sum_{\varrho \in K_{1} \cup K_{2} \cup\{1\}} \lambda_{\mu \varrho}-1} \\
\quad \times \int_{I_{\varepsilon}^{|V|}} d\left(v_{\mu}\right)_{\mu \in V} \prod_{\mu \in V} v_{\mu}^{\gamma_{\mu}^{\prime} s-\sum_{\varrho \in K_{1} \cup K_{2} \cup\{1\}} \lambda_{\mu \varrho}-1} \\
\quad \times\left(\sum_{\underline{\gamma} \leq \underline{\gamma}^{\prime}} b_{\underline{\gamma}}\left(\left(z_{\varrho}\right)_{\varrho \in K_{0}}\right) \prod_{\mu \in U} \tau_{\mu}^{\gamma_{\mu}^{\prime}-\gamma_{\mu}} \prod_{\mu \in V} v_{\mu}^{\gamma_{\mu}^{\prime}-\gamma_{\mu}}\right)^{-s}
\end{aligned}
$$

where $K_{0} \dot{\cup} K_{1} \dot{\cup} K_{2}=\{2, \ldots, r\}, \underline{\underline{\lambda}} \in \mathbb{N}_{0}^{\left(\left|K_{1}\right|+\left|K_{2}\right|+1\right)^{2}}$ regular, $U:=\{\mu \in$ $K_{1} \cup K_{2} \cup\{1\} \mid \lambda_{\mu \varrho} \geq 1$ for a $\left.\varrho \in \bar{K}_{2} \cup\{1\}\right\}, V:=\left(K_{1} \cup K_{2} \cup\{1\}\right) \backslash U$, $\varepsilon>0$ is chosen appropriately and $\widetilde{R}:(0,1)^{|U|} \rightarrow \mathbb{C}$ is continuous with $\widetilde{R}\left(\left(\tau_{\mu}\right)_{\mu \in U}\right) \ll \prod_{\mu \in U} \tau_{\mu}^{1-b}$

- Type IV:

$$
\begin{aligned}
& \sum_{\varrho} \frac{\pi}{\sin \pi \varrho}\left(-\frac{a}{\cos \Theta^{\prime}} e^{j i \Theta^{\prime}}\right)^{\varrho-1} \prod_{\mu \in V}\left(e\left(\gamma_{\mu}^{\prime} s-\lambda_{\mu 1} \varrho\right)-1\right)^{-1} \\
& \times \int_{\gamma_{0}^{\left|K_{0}\right|}} d\left(z_{\varrho}\right)_{\varrho \in K_{0}} \prod_{\varrho \in K_{0}} G_{\varrho}\left(-z_{\varrho}\right) \\
& \times \int_{(0,1)^{|U|}} d\left(\tau_{\mu}\right)_{\mu \in U} \widetilde{R}\left(\left(\tau_{\mu}\right)_{\mu \in U}\right) \prod_{\mu \in U} \tau_{\mu}^{\gamma_{\mu}^{\prime} s-\sum_{\varrho \in K_{1} \cup K_{2} \cup\{1\}} \lambda_{\mu \varrho}-1-\lambda_{\mu 1}(\varrho-1)} \\
& \times \int_{I_{\varepsilon}^{|V|}} d\left(v_{\mu}\right)_{\mu \in V} \prod_{\mu \in V} v_{\mu}^{\gamma_{\mu}^{\prime} s-\sum_{\varrho \in K_{1} \cup K_{2} \cup\{1\}} \lambda_{\mu \varrho}-1-\lambda_{\mu 1}(\varrho-1)} \\
& \times\left(\sum_{\underline{\gamma} \leq \underline{\gamma}^{\prime}} b_{\underline{\gamma}}\left(\left(z_{\varrho}\right)_{\varrho \in K_{0}}\right) \prod_{\mu \in U} \tau_{\mu}^{\gamma_{\mu}^{\prime}-\gamma_{\mu}} \prod_{\mu \in V} v_{\mu}^{\gamma_{\mu}^{\prime}-\gamma_{\mu}}\right)^{-s},
\end{aligned}
$$

where $K_{0} \dot{\cup} K_{1} \dot{\cup} K_{2}=\{2, \ldots, r\}, j \in\{ \pm 1\}, \underline{\underline{\lambda}} \in \mathbb{N}_{0}^{\left(\left|K_{1}\right|+\left|K_{2}\right|+1\right)^{2}}$ regular, $U:=\left\{\mu \in K_{1} \cup K_{2} \cup\{1\} \mid \lambda_{\mu \varrho} \geq 1\right.$ for a $\left.\varrho \in K_{2}\right\}, V:=\left(K_{1} \cup K_{2} \cup\{1\}\right) \backslash U$, $\varepsilon>0$ is chosen appropriately and $\widetilde{R}:(0,1)^{|U|} \rightarrow \mathbb{C}$ is continuous with $\widetilde{R}\left(\left(\tau_{\mu}\right)_{\mu \in U}\right) \ll \exp \left(-c \sum_{\mu \in U} \tau_{\mu}^{-1}\right)(c>0)$.

The functions which are defined by the integrals in (8.2), (8.3) and (8.5) can be continued to entire functions in $s$; the function which is defined by 
the integral in (8.4) can be continued holomorphically to the set

$$
\left\{s \in \mathbb{C} \mid \Re s>\gamma_{\mu}^{\prime-1}\left(b-1+\sum_{\varrho \in K_{1} \cup K_{2} \cup\{1\}} \lambda_{\mu \varrho}\right), \mu \in U\right\}
$$

which contains a set of the form $\left\{\Re s>b d_{1}+d_{2}\right\}$ where $d_{1}, d_{2}>0$ are constants which depend only upon the values of $\underline{\underline{\lambda}}$ and therefore only upon $P$. The functions of types I, II, III can therefore be continued meromorphically to $\Re s>b d_{1}+d_{2}$ where the poles lie in $N^{-1} \mathbb{Z}$ and have order $\leq r$; here $N \in \mathbb{N}$ depends only upon $P$. If $R \geq 1$ is chosen arbitrarily then the integral in (8.5) can be estimated uniformly in $\varrho$ and $s \in K_{R}(0)$ by $O_{R}\left(\prod_{\mu \in V} \exp \left(2 \pi \lambda_{\mu 1} \Im \varrho\right)\right)$. Define

$$
F_{R}(s)=\prod_{\mu \in V} \prod^{\left(s-s_{0}\right)}
$$

where $s_{0}$ runs through the set

$$
\left\{\lambda_{\mu 1} \varrho / \gamma_{\mu}^{\prime}+k / \gamma_{\mu}^{\prime}|| \Im \varrho\left|\leq \gamma_{\mu}^{\prime} 2 R, k \in \mathbb{Z},\right| k \mid \leq \gamma_{\mu}^{\prime} 2 R+\lambda_{\mu 1}\right\} .
$$

Then $F_{R}(s) \prod_{\mu \in V}\left(e\left(\gamma_{\mu}^{\prime} s-\lambda_{\mu 1} \varrho\right)-1\right)^{-1}$ has no poles in $K_{R}(0)$ and it can be estimated uniformly in $\varrho$ and $s \in K_{R}(0)$ by

$$
\prod_{\mu \in V} \prod_{s_{0}}\left|s-s_{0}\right| \cdot\left|e\left(\gamma_{\mu}^{\prime} s-\lambda_{\mu 1} \varrho\right)-1\right|^{-1} \ll_{R} \prod_{\mu \in V} \exp \left(-2 \pi \lambda_{\mu 1} \Im \varrho\right) .
$$

Therefore after multiplication of (8.5) by $F_{R}(s)$ one gets a series of holomorphic functions which converges uniformly on $K_{R}(0)$. (8.5) can therefore be continued meromorphically to $K_{R}(0)$ where the poles lie in

$$
\left\{N^{-1}\left(k+c_{1} \varrho\right), \ldots, N^{-1}\left(k+c_{M} \varrho\right) \mid k \in \mathbb{Z}, \varrho \text { a nontrivial zero of } \zeta\right\}
$$

and are of order $\leq|V| \leq r$. Here $N \in \mathbb{N}$ and $c_{1}, \ldots, c_{M} \in \mathbb{N}_{0}$ are constants which depend only upon $P$. As $R \geq 1$ may be chosen arbitrarily (8.5) can be continued meromorphically to $\mathbb{C}$ with poles of order $\leq r$ which lie in (8.6). Consequently, $S(s)$ can be continued meromorphically to $\Re s>b d_{1}+d_{2}$ with poles of order $\leq r$ which lie in (8.6). If $N^{-1}\left(k+c_{m} \varrho\right)$ is a pole of $S(s)$ then its real part is $\leq \sigma_{\mathrm{a}}^{*}$ and therefore $k \leq\left[N \sigma_{\mathrm{a}}^{*}\right]=: c$. As $b \in \mathbb{R}^{-} \backslash \mathbb{Z}$ can be chosen arbitrarily the statement of the theorem follows.

\section{References}

[1] P. Cassou-Noguès, Valeurs aux entiers négatifs des séries de Dirichlet associées à un polynôme I, J. Number Theory 14 (1982), 32-64.

[2] H. Davenport, Multiplicative Number Theory, Markham, 1967.

[3] J. Jorgenson and S. Lang, Basic Analysis of Regularized Series and Products, Lecture Notes in Math. 1564, Springer, 1993. 
[4] B. Lichtin, Generalized Dirichlet series and b-functions, Compositio Math. 65 (1988), 81-120.

[5] - The asymptotics of a lattice point problem associated to a finite number of polynomials I, II, Duke Math. J. 63 (1991), 139-192; 77 (1995), 699-751.

[6] K. Mahler, Über einen Satz von Mellin, Math. Ann. 100 (1928), 384-395.

[7] Hj. Mellin, Eine Formel für den Logarithmus transcendenter Functionen von endlichem Geschlecht, Acta Soc. Sci. Fenn. 29 (1900), no. 4.

[8] -, Die Dirichlet'schen Reihen, die zahlentheoretischen Funktionen und die unendlichen Produkte von endlichem Geschlecht, Acta Math. 28 (1904), 37-64.

[9] P. Sargos, Prolongement méromorphe des séries de Dirichlet associées à des fractions rationnelles de plusieurs variables, Ann. Inst. Fourier (Grenoble) 34 (3) (1984), 83-123.

[10] -, Croissance de certaines séries de Dirichlet et applications, J. Reine Angew. Math. 367 (1986), 139-154.

[11] G. Tenenbaum, Introduction à la théorie analytique et probabiliste des nombres, Publ. Inst. Elie Cartan, Université de Nancy, 1990.

Mathematisches Institut

Albert-Ludwigs-Universität

Eckerstr. 1

D-79104 Freiburg, Germany

E-mail: peter@arcade.mathematik.uni-freiburg.de 